\title{
Identification of TNFRSF4 as a Diagnosis and Prognosis Biomarker Associated with Immune Microenvironment in Uterine Corpus Endometrial Carcinoma
}

\section{Heng Ma}

Peking Union Medical College Hospital

\section{Penghui Feng}

Peking Union Medical College Hospital

Shuangni Yu

Peking Union Medical College Hospital

\section{Ruiqin Han}

Peking University School of Basic Medical Sciences

\section{Zaixin Guo}

Peking Union Medical College Hospital

Qi Yu ( $\square$ yuqi2008001@sina.com)

Peking Union Medical College Hospital https://orcid.org/0000-0001-9737-5957

Jie Chen

Peking Union Medical College Hospital

\section{Research Article}

Keywords: TNFRSF41, biomarker2, prognosis3, tumor microenvironment4, uterine corpus endometrial carcinoma5

Posted Date: May 17th, 2021

DOl: https://doi.org/10.21203/rs.3.rs-488368/v1

License: (c) (i) This work is licensed under a Creative Commons Attribution 4.0 International License. Read Full License 


\section{Abstract}

\section{Background}

The interaction between tumor microenvironment (TME) and tumors offers various targets in mounting anti-tumor immunotherapies. However, the diagnostic and prognostic biomarkers in uterine corpus endometrial carcinoma (UCEC) are still limited. Here, we aimed to analyze the TME features and identify novel prognostic biomarkers for UCEC.

\section{Methods}

ESTIMATE, CIBERSORT, protein-protein interaction (PPI) network, univariate Cox regression, and functional enrichment analysis were performed to identify immune- and survival-related hub genes as well as possible molecular mechanisms. The limma package and the deconvolution algorithm were adopted to estimate the tumor-infiltrating immune cells (TICs) abundance and their relationship with the target gene. Tissue microarrays (TMAs) of UCEC were evaluated to validate protein expression of the identified immune markers, including TNFRSF4, CD4, and CD8. The receiver operating characteristic (ROC) curve was used to determine the efficacy of TNFRSF4 in diagnosing UCEC.

Results

Two genes, TNFRSF4 and S1PR4, were screened out from 386 intersection differential expression gene (DEGs) shared by ImmuneScore and StromalScore in UCEC. Highlighted by TNFRSF4, we found that it was not only positively correlated with the TICs (mainly $\mathrm{CD} 4^{+} \mathrm{T}$ cells, $\mathrm{CD} 8^{+} \mathrm{T}$ cells, and Tregs) but significantly related to diagnosis and prognosis in patients of UCEC, both verified by data from the TCGA database and clinical samples.

\section{Conclusions}

Collectively, TNFRSF4 could serve as a high-profile biomarker to robustly predict immune microenvironment, clinical diagnosis and prognosis for UCEC.

\section{Background}

Uterine corpus endometrial carcinoma (UCEC), as the second most common carcinoma of the female genital tract globally ${ }^{[1]}$, is characterized by abnormal vaginal bleeding or discharge, especially for postmenopausal women, abdominal pain, and other clinical signs or symptoms. According to estimates in 2020 , there will be 65,620 new cases of UCEC diagnosed, 12,590 deaths caused by UCEC in the United States ${ }^{2}{ }^{2]}$, and both morbidity and mortality rates have exhibited a sustained rise, particularly in cases among women under the age of 50 years (1.3\% per year from 2007-2016) [3]. The common risk factors include continuous exposure to increased estrogen due to diet, diabetes or obesity, age, tamoxifen, Lynch

syndrome, and the abnormal onset of menarche or menopause ${ }^{[4-7]}$. With early diagnosis and treatment 
for uterine corpus cancer, the five-year survival rate could achieve $80 \%$, approximately ${ }^{[8]}$. In clinical findings, all the survivors from the early stage are confronted with enormous risks for metastasis or recurrence. Once it happened, there is, in most cases, only less than four months left for the patients despite that the advanced therapeutic patterns such as neoadjuvant chemotherapy, radiotherapy, and target treatment are available ${ }^{[9]}$.

The tumor microenvironment (TME) is necessary for the living of tumor cells. It, either directly or indirectly, affects tumor occurrence and development through maintaining a quiescent state of immune contexture ${ }^{[10]}$, promoting tumor angiogenesis ${ }^{[11]}$, changing biological features of carcinoma ${ }^{[12]}$, or even regulating the cancer stem cell activity ${ }^{[13,14]}$. The orchestration of these changes not only engenders tumor survival and expansion by establishing a suitable TME but creates heterologous cell types within tumors by giving tumor selection for mutations ${ }^{[15]}$. Additionally, TME is inextricably linked to tumor immune suppression or activation. In addition to tumor cells, the stromal cells, chemokines, and cytokines, TME comprises innate immune cells (including macrophages, neutrophils, dendritic cells, myeloid-derived suppressor cells, and natural killer cells) and adaptive immune cells (including T cells and $B$ cells) ${ }^{[16]}$. Both populations of immune cells innately modulate tumor cell-intrinsic and extrinsic processes within TME. To date, much work has been devoted to establishing moderate-resolution TME data for the analysis and characterization of the cross-talk between the tumor and its microenvironment, such as immunohistochemistry (IHC) and bulk tissue microarrays (TMAs) ${ }^{[17,18]}$. ESTIMATE (Estimation of Stromal and Immune cells in Malignant Tumor tissues using Expression data) is a highly rated tool developed by Kosuke Yoshihara and his co-workers for predicting tumor purity mainly based on the sSGSEA algorithm ${ }^{[19]}$, which has helped us to estimate the abundance of tumor-infiltrating lymphocytes and subsequently stratify patients for predicting clinical outcomes ${ }^{[20]}$. Increasing evidence has elucidated that the difference in the efficacy of tumor immunotherapy was mainly due to the heterogeneity of TME ${ }^{[21]}$. Even though most of these valuable methods and theories have been well applied and tested in many cancers, many works are still needed to make sense of the correlation between TME biological characteristics and the aberrant expression of immune-related genes in UCEC. Therefore, more reliable and effective surrogate biomarkers are left to be explored to predict the pathological behavior of UCEC and enable the stratification of patients according to immune-related criteria for improving prognostic and selecting appropriate adjuvant therapy to guide clinical decisions.

Here, we used the ESTIMATE algorithms and the tumor-infiltrating immune cells (TICs) profile to perform a comprehensive analysis of TME and detect related gene expression in patients with UCEC, through which TNFRSF4 was identified to be associated with the diagnosis and prognosis of UCEC. Moreover, to support our findings, clinical specimens were applied to validate the expression of TNFRSF4 in UCEC and adjacent normal tissues. The correlations of TNFRSF4 with clinicopathologic features, immune-related markers (including CD4 and CD8) were evaluated either. Overall, our results indicated that TNFRSF4 might be a diagnostic- and prognostic-valued biomarker, as a crucial role in TME of UCEC.

\section{Methods}


Transcriptome RNA-seq data of 587 UCEC cases (552 tumor samples and 35 controls) and corresponding clinical information were obtained from the TCGA database (https://portal.gdc.cancer.gov/).

\section{Estimation Evaluation and DEGs Generation}

Estimate algorithm was applied to determine the immune-stromal component in TME of each sample utilizing estimate package in R software (https://r-forge.r-project.org/), the final results of which were calculated as StromalScore, ImmuneScore, and ESTIMATEScore, representing the ratio of stromal cell, immune cell, and the summation of both cells, respectively ${ }^{[22]}$. The higher scores correspondingly indicated more elevated composition abundance. Based on the median of the StromalScore and ImmuneScore, samples were divided into either the high or low score groups, and survival analysis was carried out by R software loaded with survival and survminer packages ${ }^{[23]} .541$ tumor samples were finally analyzed with complete survival information, and the survival curve was produced by the KaplanMeier method and compared between groups by the log-rank test. At the same time, clinical data were acquired and analyzed to ascertain the correlation between scores and clinical parameters, including age and grade. Then, differentially expressed genes (DEGs) of StromalScore and ImmuneScore between the high and low score groups were screened out by limma package ${ }^{[24]}$ with false discovery rate $(F D R)<0.05$ and $\mid \log$ fold change $(\mathrm{FC}) \mid>1$.

\section{Functional Enrichment Analysis}

DEGs were displayed and processed as heatmaps according to the expression level using the pheatmap package for clustering (https://CRAN.R-project.org/package=pheatmap). To determine the functional enrichment for gene ontology (GO) and Kyoto Encyclopedia of Genes and Genomes (KEGG) pathways analysis, R packages (clusterProfiler, enrichplot, org.Hs.eg.db, and ggplot2) were utilized to identify gene functions and achieve visualization for functional profiles ${ }^{[25,26]}$, where q-value of less than 0.05 were considered significantly enriched.

PPI Network Construction and Cox Regression Analysis

Protein-protein interaction (PPI) network was produced through the online analytical database, STRING (https://string-db.org/cgi/input.pl) to clarify the interactive relation among genes (only were the edges and nodes kept when the confidence of interactive relationships $>0.4$ ). To further confirm the highly interconnected genes, the MCODE plugin of Cytoscape (https://cytoscape.org/) was applied, and the interactive hub cluster was selected with 56 hub genes and 794 edges (degree cutoff $=0.2, \mathrm{~K}$-core $=2$ ). Additionally, Cox regression analysis was conducted by using R package survival. Genes with $P$-value < 0.05 were displayed.

Gene Set Enrichment Analysis 
H.all.v7.2.symbols Hallmark and C7.all.v7.2.symbols immunological signatures gene sets were downloaded from the Molecular Signatures Database

(http://software.broadinstitute.org/gsea/msigdb/index.jsp). All gene sets were analyzed utilizing the software of GSEAS-4.0 downloaded from Broad Institute.

\section{TICs Profile}

The limma package in R was applied to normalize the data to evaluate the proportion of TICs, and then a standardized gene expression profile was uploaded to CIBERSORT. The deconvolution algorithm was adopted to estimate the TIC abundance ${ }^{[27]}$. Only 235 tumor samples with $P$-value $<0.05$ were screened out by quality filtering and applied to the following analysis.

Tissue Microarray and Ethical Statement

Commercialized TMAs were purchased from Shanghai Outdo Biotech Co. Ltd. (panel HUteA020CS01, HUteA045PG01, and HUteA060CS01). These TMAs, composed of 121 cores (85 carcinomas and 36 adjacent tissue, $2 \mathrm{~mm}$ in diameter ), were accessed from 85 patients with pathology grade $\mathrm{I}-\mathbb{Z}$ endometrioid adenocarcinoma, aged between 35 to 80 . The research scheme of this study has been reviewed and approved by the Ethics Committee of Peking Union Medical College Hospital (ethics, SK973), and following the guidelines approved by the Institutional Review Board of our hospital.

Immunohistochemistry $(\mathrm{IHC})$ and evaluation of immunostaining

IHC staining was performed as described previously ${ }^{[28,29]}$. Briefly, TMA specimens were deparaffinized, hydrated in xylol and ethanol, respectively, and subjected to heat mediated antigen retrieval with Tris/EDTA buffer (PH 9.0) at $95^{\circ} \mathrm{C}$ for $10 \mathrm{~min}$. Endogenous peroxidase activity was quenched by $0.3 \%$ hydrogen peroxide diluents before commencing with the $\mathrm{IHC}$ staining protocol. After blocking with normal goat serum (ZLI-9056, ZSGB-BIO, China), TMA sections were incubated with antibodies against TNFRSF4 (ab264465, 1:1000; Abcam), CD8A (encoding CD8) (85336S, 1:100; CST), and CD4 (4B12, 1:100; Leica), coated at $4{ }^{\circ} \mathrm{C}$ overnight, and with biotin-conjugated secondary reagents for $30 \mathrm{~min}$. Human tonsil and placenta tissues were used as the positive/negative control with/without primary antibodies. At the end of the staining, whole TMA slides were digitally scanned at $\times 400$ using a NanoZoomer S360 (Hamamatsu, Japan) for visualization.

This study was conducted according to the Reporting Recommendations for Tumor Marker Prognostic Studies (REMARK) guidelines ${ }^{[30]}$. To evaluate the immunostaining, two experienced pathologists were blinded to the patients' clinical outcomes and independently determined the scores using the semiquantitative immunoreactive Score by Remmele and Stegner ${ }^{[31]}$. Given that TNFRSF4, CD 8 and CD4 were expressed in tumor-infiltrating lymphocytes (TILs), its scoring has used the method of Erminia Massarelli ${ }^{[32]}$ by counting positive cells in the five random square areas at $400 \times$ magnification, and the expression of each marker was recorded as the density of positive cells $/ \mathrm{mm}^{2}$. 
SPSS (Version. 22, Chicago, IL, USA) was used for statistical analyses. Shapiro-Wilk test, combined with normality plots, was applied to determine the normal distribution. For unpaired samples, comparisons between two groups were analyzed by the Mann-Whitney test or Student's t-test. As for paired samples, the Wilcoxon rank-sum test was applied. Besides, multiple comparisons were carried out based on the Kruskal Wallis rank-sum test to compare the difference among groups. The two-sided Person chi-squared test or Spearman rank correlation test was used to evaluate the relationship between TNFRSF4 IHC scores and immune-related gene expression levels. The Kaplan-Meier analysis by the log-rank test and univariate Cox regression analysis were performed to assess survival and estimate the independent prognostic factors. Statistical significance was defined when $P$-value was less than 0.05 .

\section{Results}

Research Thoughts and Structure of This Study

The analysis process of our study was displayed in the flow chart (Fig. 1). On the whole, in order to evaluate the abundance of TICs and the component ratio of immune and stromal cells in UCEC samples, 587 cases of transcriptome RNA-seq data from the TCGA database, including 35 normal tissues and 552 tumor ones, were retrieved and processed by CIBERSORT and ESTIMATE algorithms. 386 DEGs shared by ImmuneScore and StromalScore were determined, which were further analyzed to screen out the hub network genes based on PPI network and MCODE scores. Meanwhile, univariate Cox regression was performed to filtrate out prognosis-related genes. Then, target genes were finally intersected and obtained by genes from the core cluster in the PPI network and the survival-correlated genes from univariate Cox regression analysis. TNFRSF4 and S1PR4 were determined, and we placed emphasis mainly on TNFRSF4 for the subsequent series of investigations. Then, we confirmed the prognostic significance and indicative role in TME remodeling of TNFRSF4 in UCEC, as well as the correlation of TNFRSF4 with the abundance of TICs.

Clinicopathologic Significance of the Estimate Scores

To reveal the relationship between the component of immune or stromal cells and the clinical outcomes, survival analysis was performed for ImmuneScore, StromalScore, and ESTIMATEScore, respectively. As known, the higher score estimated in ImmuneScore or StromalScore indicated a higher proportion of corresponding immune or stromal cells in TME. Besides, ESTIMATEScore represented the sum of ImmuneScore and StromalScore; in other words, the higher the score, the lower the purity of the tumor. As presented in Fig. 2A, the percentage of immune components was positively correlated with the overall survival rate despite that StromalScore and ESTIMATEScore made no difference (Fig. 2B, C). These results manifested that the immune composition in TME was a more reliable indicator of the prognosis for UCEC patients. To further estimate the correlation between the three scores and clinicopathologic parameters. We compared patients, groups by age or pathological grade of UCEC. Our results demonstrated that tumor of UCEC patients over 65 years old had lower StromalScore and 
ESTIMATEScore than younger patients $(P<0.01$ and $<0.05$, respectively) even though ImmuneScore did not differ in terms of age as shown in Fig. 3A, C, E. As for the pathological grade, it was worth noting that our data exhibited consistently decreasing trends in the three scores as tumor progressed even though not all comparisons among groups with different grade were statistically significant (Fig. 3B, D, F). Intriguingly, groups of grade $\nabla$ showed lower ImmuneScore, StromalScore and ESTIMATEScore $(P=0.013$,

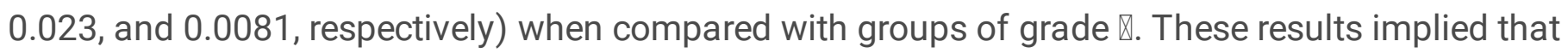
advanced age ( $>65$ ) and higher grade of UCEC, especially grade $\otimes$, tended to had less immune and stromal component, possibly signified poor prognosis.

DEGs Shared by ImmuneScore and StromalScore and Functional Enrichment

In order to explore the variation of gene profile in the wake of the alteration of the immune or stromal component in TME, we compared the expression level of genes between the high- and low-score groups. Among them, 716 DEGs were finally determined regarding ImmuneScore, including 552 up-regulated genes and 164 down-regulated genes. Similarly, there were 731 DEGs screened out from StromalScore, which mainly consisted of highly expressed genes. As shown in the heatmaps, the top 50 DEGs were displayed based on ImmuneScore and StromalScore, and the gene expression pattern showed an apparent difference between groups (Fig. 4A, B). After the intersection, 386 genes were shared in common by ImmuneScore and StromalScore, with 366 genes were up-regulated and the rest down-regulated (Fig. 4C, D). Then, functional enrichment analysis was carried out based on these overlapped genes. Our results indicated that the identified genes were chiefly enriched in the regulation of lymphocyte activation, differentiation, and proliferation, as well as regulation of leukocyte cell-cell adhesion in the biological process (BP). About the cellular component (CC) of GO analysis, these genes encoding proteins were the main components of the external side of the plasma membrane, immunological synapse, granule membrane, and protein complex involved in cell adhesion. As for molecular function (MF), the DEGs prevailingly enrolled in immune or cytokine receptor activity and chemokine binding or activity (Fig. 4E). KEGG analysis revealed that chemokine signaling pathway and cytokine-cytokine receptor interaction were most relevant to these DEGs (Fig. 4F). It thus appeared that the overall function of these DEGs primarily focused on immune-related activities, which essentially suggested that the involvement of immune modulation was a notable feature of TME in UCEC.

Intersection Analysis of PPI Network and Cox Regression

So as to elucidate the underlying mechanism, we explored the PPI network constructed by the STRING database, and thus, the interaction between genes was identified. On this basis, the MCODE plugin of Cytoscape software was applied to seek the hub gene cluster. Finally, 56 genes were identified with 794 edges among these genes, displayed in Fig. 5A, and the bar plot in Fig. 5B showed the top 40 genes, ranked by the number of adjacent nodes. Additionally, to identify prognosis-related genes among 386 DEGs in UCEC, univariate Cox regression analysis was performed, and the results manifested that the expression level of 16 genes was highly correlated with the survival outcome as shown in the forest plot 
(Fig. 5C). Subsequently, two genes, TNFRSF4 and S1PR4, were screened out after intersection analysis based on the above PPI network and univariate Cox regression analysis (Fig. 5D).

\section{Prognostic Significance and Indicative Role in TME Remodeling of TNFRSF4 in UCEC}

According to previous studies on UCEC, we found a gene, namely TNFRSF4, barely reported on UCEC but was a high-profile target on other cancers. TNFRSF4 was first discovered on the surface of activated $\mathrm{CD}^{+} \mathrm{T}$ cells in rats, which belonged to tumor necrosis factor receptor family member ${ }^{[33]}$. TNFRSF4 played a vital role in immune regulation in multiple cancers as a crucial immune checkpoint. For example, it was once proved to be subjected to forkhead box P3 (Foxp3) to reduce the immunosuppression action of Tregs in breast cancer ${ }^{[34]}$. Similarly, TNFRSF4 also exhibited a promising future in targeted therapy towards other tumors such as gastric carcinoma, leukemia, and squamous cell carcinoma of the head and neck ${ }^{[35-37]}$. In the following analysis, our data indicated that TNFRSF4 showed higher expression level in tumor tissues than normal tissues either in unpaired or paired samples $(P<0.001$ and $P=0.028$, respectively), as shown in Fig. $6 \mathrm{~A}, \mathrm{~B}$. Interestingly, the expression level decreased as aged when compared with the younger group (Fig. 6C). Similarly, tumors of grade $\nabla$ or $\nabla$ exhibited a consistently less amount of TNFRSF 4 when compared with tumors of grade $\otimes$ (Fig. 6D). More importantly, the tumor samples were classified into two groups, the high or low-expression groups, according to the median expression of TNFRSF4 for survival analysis. Here, we found that increased expression of TNFRSF4 was significantly correlated with survival outcome (Fig. 6E). These results signified that TNFRSF4 seemed to act as a protective factor in UCEC; however, this so-called protection appeared to decline with aging and tumor progression inevitably. Considering that the expression of TNFRSF4 was positively related to the survival rate and negatively correlated with the age or grade of UCEC patients, GSEA was implemented for the high and low-expression groups of TNFRSF4. As shown in Fig. 6F, the genes in the TNFRSF4 highexpression group were chiefly enriched in immune-related activities, such as IFN- $y$ response, IL-2/STAT5 signaling and IL-6/JAK/STAT3 signaling. In terms of the TNFRSF4 low-expression group, the genes were mainly involved in the G2M checkpoint, mitotic spindle, $m$ TORC1 signaling and protein secretion (Fig. 6G). For C7 collection defined by MSigDB, the immunologic gene sets, multiple immune functional gene sets enrolled in the high and low TNFRSF4 expression groups (Fig. $6 \mathrm{H}, \mathrm{I})$. These results suggested that TNFRSF4 might be a potential indicator for the status of TME.

\section{Correlation of TNFRSF4 with the Abundance of TICs}

Given the above findings, the component of TICs of each sample was further estimated using the CIBERSORT algorithm to detect the pertinence relation of TNFRSF4 expression with the immune microenvironment and 22 kinds of immune cell profiles in UCEC samples were identified, as shown in the bar plot (Fig. 7A). It was observed that immune cells in UCEC were mainly composed of T cells and macrophagocytes. Besides, the correlation among the immune cells was also displayed. The results showed that the proportion of $\mathrm{CD} 8^{+} \mathrm{T}$ cells was negatively correlated with the presence of $\mathrm{CD} 4^{+}$memory resting $\mathrm{T}$ cells and M0 macrophagocytes (correlation coefficient $=-0.55$ or -0.63 , respectively). Conversely, 
the content of $\mathrm{CD} 8^{+} \mathrm{T}$ cells was positively related to the scale of $\mathrm{CD} 4^{+}$memory activated $\mathrm{T}$ cells, as seen in Fig. 7B.

To verify the majors immune cells affected by the expression of TNFRSF4, the difference and correlation analysis were carried out and results demonstrated that a total of 5 kinds of TICs differed between the high and low-TNFRSF4 expression groups, including $\mathrm{CD} 8^{+} \mathrm{T}$ cells, regulatory $\mathrm{T}$ cells, resting dendritic cells, eosinophils, and neutrophils (Fig. 8A). Furthermore, seven kinds of immune cells were correlated with the expression of TNFRSF4 (three positively and four negatively), as observed in Fig. 8B. Finally, four types of immune cells were identified to be vitally interconnected with TNFRSF4 in view of the differential expression and degree of relevance via intersection analysis (Fig. 8A). These results further hinted that the expression of TNFRSF 4 had much to do with the immune activity of TME in UCEC.

Identification of the Expression, Correlation, and Diagnostic Performance of TNFRSF4

From the TCGA mRNA expression profiles, we found that the expression of TNFRSF4 was positively correlated with $C D 4(\mathrm{R}=0.51)$ and $C D 8 A(\mathrm{R}=0.47)(F i g .9 \mathrm{~A}, \mathrm{~B})$. In IHC staining, the proteins of TNFRSF4, CD4, and CD8 were over-expressed in UCEC tissues (mainly on tumor immune infiltrating cells) compared with normal tissues or even paired adjacent normal tissues (Fig. 9E, F). Moreover, these proteins exhibited a cytoplasmic and membranous staining pattern in the UCEC samples, which were consistent with the results in non-small cell lung cancer ${ }^{[32]}$. Representative images of TNFRSF4, CD4, and CD8 were shown in Fig. 9D. However, no statistical correlation was found between TNFRSF4 expression and clinicopathologic parameters, such as age and histological grade (Table 1). 
Table 1

Association among TNFRSF4 expression and clinicopathologic parameters, CD 4 or CD8 in patients with UCEC of the validation cohort.

\begin{tabular}{|c|c|c|c|}
\hline \multirow[t]{2}{*}{ Parameters } & \multicolumn{2}{|c|}{ TNFRSF4 expression } & \multirow[t]{2}{*}{$P$-value } \\
\hline & High & Low & \\
\hline \multicolumn{3}{|l|}{ Age (years) } & 0.315 \\
\hline$\leq 50$ & $11(61.1)$ & 7(38.9) & \\
\hline$>50$ & $32(47.8)$ & $35(55.2)$ & \\
\hline \multicolumn{3}{|l|}{ Histological grade } & 0.709 \\
\hline I & $16(53.3)$ & $14(46.7)$ & \\
\hline II-III & $27(49.1)$ & $28(50.9)$ & \\
\hline \multicolumn{3}{|l|}{ CD4 } & $<0.01$ \\
\hline High & $28(65.1)$ & $14(34.9)$ & \\
\hline Low & 15(33.3) & $28(66.7)$ & \\
\hline \multicolumn{3}{|l|}{ CD8 } & $<0.001$ \\
\hline High & $30(73.2)$ & 11(26.8) & \\
\hline Low & 13(29.5) & $31(70.5)$ & \\
\hline \multicolumn{4}{|c|}{ Data were expressed as number (Percentage). } \\
\hline \multicolumn{4}{|c|}{ Chi-square tests or Fisher's exact test for categorical variables } \\
\hline
\end{tabular}

To assess the diagnostic value of TNFRSF 4 in UCEC, the ROC diagnosis model was performed. Surprisingly, we uncovered that TNFRSF4 had a higher diagnostic significance in either the TCGA dataset (the AUC value $=0.715$ ) or clinical specimens (the AUC value $=0.777)($ Fig. 9C, G). Altogether, these data implied the potential role of TNFRSF4 in immune microenvironment remodeling and diagnostic performance for UCEC.

\section{Discussion}

Given the limited understanding of the details of TME for UCEC, especially the complicated and volatile characteristics of the immunological microenvironment, in this study, we comprehensively analyzed the alteration of TME and the composition of TICs in UCEC based on CIBERSORT algorithms. We then determined TNFRSF4 and SIRP4 as immune- and prognosis-related hub genes obtained by the intersection between Cox proportional hazards regression and PPI analysis. Finally, focused on TNFRSF4, we found that TNFRSF4 might be responsible for stably maintaining the immunomodulatory 
characteristics of TME, thus leading to a better prognosis, which was further validated in the TCGA dataset and patient cohort.

Exploring TME signature genes is a necessary step in uncovering intricate relationships among clinical features and molecular characteristics. ESTIMATE algorithm, as a classical tool, has been used to screen out potential novel biomarkers based on stromal and immune gene sets from each sample in the tumor expression matrix. Here, our data demonstrated that ImmuneScore had a more significant correlation with the overall survival rate than StromalScore and ESTIMATEScore, which suggested that immune microenvironment composition was more critical in modulating cancer progression and prognosis of UCEC patients. Besides, the correlation between the three scores and clinicopathologic parameters (age, histological, and grade) revealed that older patients with UCEC of higher grade showed more unfavorable clinical outcomes. This phenomenon could be explained that lower immune cell composition or less 'immune driven' genotype tended to appear in patients with advanced cancer ${ }^{[38]}$. By the way, Peigen Chen et al. demonstrated that the histological type extracted from the TCGA database was associated with immune and stromal scores in spite that both of which made no difference ${ }^{[39]}$.

To screen out novel genes related to TME in UCEC, 587 UCEC cases from the TCGA database were divided into the high and low-score groups based on the median of the StromalScore and ImmuneScore. Our results showed that 386 DEGs were shared in common by ImmuneScore and StromalScore, with 366 genes were up-regulated and 20 down-regulated. Next, to clarify the functional enrichment of these overlapped genes, GO terms and KEGG were used. In this part, we found that most of the intersection genes were chiefly involved in immune modulation, including $T$ cell activation, regulation of lymphocyte activation, immune receptor activity, and chemokine signaling pathway. Based on these results, it suggested that these DEGs played a vital role in modulating the immunological environment, and implied series of intricate interactions among non-neoplastic cells within the TME could impact tumor fate ${ }^{[16]}$.

To identify potential biomarkers, Cox regression and PPI network analysis were carried out in the present study. We identified two genes, TNFRSF4 and S1PR4, after intersection analysis based on the top 40 PPI genes cluster and 16 prognostic-related genes. Of them, TNFRSF4, also known as OX40 or CD134, a costimulatory molecule mainly expressed on $\mathrm{CD} 4^{+}$and $\mathrm{CD} 8^{+} \mathrm{T}$ cells, which belongs to the TNF-receptor superfamily ${ }^{[40]}$, has been selected for further investigation. Initially, evidence was brought by Paterson, D.J. ${ }^{[41]}$, who identified TNFRSF4 as a specific marker of T-cell activation and survival when cross-linked with its ligand TNFSF4 ${ }^{[41,42]}$. Then emerging evidence highlighted that TNFRSF4 was a promising therapeutic target for T cell-mediated anti-tumor immunotherapy $[37,43,44]$. However, there are still some contradictory sides of TNFRSF4, especially that the prognostic effects of TNFRSF4 among different kinds of tumors are often poles apart. These paradoxical results could be explained by heterogeneous TME and homeostatic regulation of Tregs ${ }^{[45]}$. Encouragingly, the latest evidence reinforced the notion that immunotherapy with TNFRSF4 agonists would trigger a new trend in cancer therapies, and there was no sanitary approval for TNFRSF4 agonists up to now ${ }^{[46]}$. For example, TNFRSF4 stimulation plus a CTLA-4 blockade functioned as a potential therapeutic strategy to eradicate disseminated tumors by depleting 
tumor-specific Tregs within the tumor-infiltrating lymphocytes in tumor-bearing mice ${ }^{[47]}$. In short, TNFRSF4 might act as a win-win path to reestablish T-cell antitumor activity [48].

Intriguing, as a second-generation immune checkpoint molecule, there are relatively few studies on UCEC, but it was extremely favored in T cell-mediated anti-tumor immunotherapy in other cancers. It encourages us to explore whether TNFRSF4 can serve as a reliable prognostic biomarker. In our investigation, we found that an elevated expression of TNFRSF4 indicated a better prognosis but was negatively correlated with age or grade in UCEC patients from the TCGA database. Furthermore, GSEA findings revealed that transcriptome signatures of immune-related activities were positively correlated with TNFRSF4 and chiefly enriched in inflammatory signaling pathways (such as IFN- $y$ response) in the TNFRSF4 overexpressed group. To our knowledge, IFN- $\gamma$ is a pleiotropic molecule with widely involved pro- and antitumorigenic effects ${ }^{[49]}$, and $C D 8^{+} \mathrm{T}$ lymphocyte-mediated anti-tumor immunity relies on IFN-y production for $C D 8^{+} T$ cells expansion ${ }^{[50]}$. At the same time, related signal pathways (e.g. G2M checkpoint, mitotic spindle, and $m T O R C 1$ signaling) that regulated tumor cell proliferation and survival were mainly enriched in the TNFRSF4 low-expression group. Accordingly, the low expression of TNFRSF4 might promote the progression of the tumor to some extent in the UCEC. Of course, further studies are needed to verify this speculation in vivo and in vitro.

Immune cell infiltration has held great promise as a new biomarker of prognosis in patients with different types of solid tumors, including endometrial cancer [51], liver cancer ${ }^{[52]}$, colon cancer ${ }^{[53]}$, brain tumor ${ }^{[54]}$, and pancreatic ductal adenocarcinoma ${ }^{[55]}$. To further investigate the role of TNFRSF4 in TME, we firstly estimated the component of TICs in UCEC samples by the CIBERSORT algorithm. Consistent with previous studies, our results demonstrated that $\mathrm{T}$ cells and macrophagocytes were dominant in TME. The proportion of $\mathrm{CD} 8^{+} \mathrm{T}$ cells was positively related to the amount of $\mathrm{CD} 4^{+}$memory-activated $\mathrm{T}$ cells rather than $\mathrm{CD} 4^{+}$memory resting $\mathrm{T}$ cells and $\mathrm{M} 0$ macrophagocytes. As known, $\mathrm{CD} 4^{+} \mathrm{T}$ cell help is indispensable for sustaining $\mathrm{CD}^{+} \mathrm{T}$ cell function during chronic viral infection and engaged in antitumor activities by

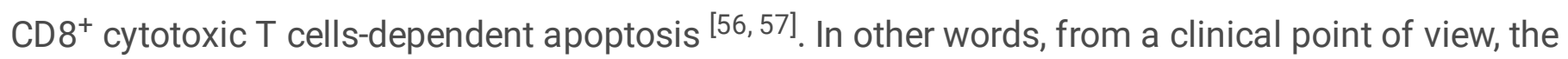
presence of immune cell infiltration with high levels of cytotoxicity $C D 8^{+} \mathrm{T}$ cells signified a favorable prognosis in cancers. Moreover, our study figured out that the expression of TNFRSF4 was positively correlated with $\mathrm{CD} 4^{+} \mathrm{T}$ cells, $\mathrm{CD} 8^{+} \mathrm{T}$ cells, and Tregs by bioinformatics analysis. Notably, we also found this relationship in UCEC via TMA validation except for Tregs. Based on our results, further confirming this correlation and evaluating the prognostic significance of TNFRSF4 in large-scale cohort studies are of great importance.

Inspired by these results, to further verify the protein expression of TNFRSF4 in clinical specimens, commercialized TMAs, consisted of 85 UCEC patients (including 36 paired cases), were validated. Our result showed that TNFRSF4 was overexpressed in cancer tissues when compared with paired adjacent normal tissues, and it was mainly located in the TILs within the tumor stroma. It's worth noting that the distinct location of TNFRSF4, as previously described, indicated different prognostic value. For example, the expression of TNFRSF4 on TILs was correlated with favorable prognosis in human cancers, including 
advanced gastric cancer, non-small cell lung cancer, ovarian carcinoma, malignant melanoma, and

colorectal cancer ${ }^{[32,58-61]}$, but the expression on cancer cells was associated with a poorer outcome including hepatocellular carcinoma and cutaneous squamous cell carcinoma ${ }^{[62,63]}$. Furthermore, TNFRSF4-positive cells were also observed to interact with $\mathrm{CD} 4^{+}$and $\mathrm{CD} 8^{+} \mathrm{T}$ cells in our study, which would be due to the role of TNFRSF 4 in the proliferation of $C D 4^{+}$and $C D 8^{+} T$ cells, as well as the survival of antigen-specific memory $T$ cells ${ }^{[64-66]}$. Additionally, one unexpected result by ROC curve analysis suggested that TNFRSF4 expression could serve as a potential indicator of favorable diagnosis among patients with UCEC, and this diagnosis performance was better than both CD4 and CD8.

Our current study has limitations. First, given the inherent biases produced by TMAs, those results must be interpreted cautiously and improved in future studies. Second, the coexpression and function of TNFRSF4 within the component of TME are undefined; multiplexed quantitative immunofluorescence is essential to make up for it. Apart from the above limitations, the clinical prognostic value of TNFRSF4 was not validated in cohorts with UCEC patients as well, but these endeavors are underway in our team.

\section{Conclusions}

In summary, we identified a novel molecule in UCEC, TNFRSF4, and an elevated expression of it tends to signify favorable clinical outcomes, closely related to the abundance of $\mathrm{CD} 4^{+}$and $\mathrm{CD} 8^{+} \mathrm{T}$ cells. As an immune-related gene, it held feasible and reliable diagnostic and prognostic management in patients with UCEC. Notably, our study suggested that a deep understanding of TNFRSF4 might unravel a clue for the optimal chance of therapeutic success against UCEC.

\section{Abbreviations}

Uterine corpus endometrial carcinoma (UCEC), tumor microenvironment (TME), immunohistochemistry (IHC), tissue microarrays (TMAs), tumor-infiltrating immune cells (TICs), differentially expressed genes (DEGs), false discovery rate (FDR), fold change (FC), gene ontology (GO), Kyoto Encyclopedia of Genes and Genomes (KEGG), protein-protein interaction (PPI), tumor-infiltrating lymphocytes (TILs), biological process (BP), cellular component (CC), molecular function (MF).

\section{Declarations}

\section{Ethics approval and consent to participate}

The research scheme of this study has been reviewed and approved by the Ethics Committee of Peking Union Medical College Hospital (ethics, S-K973), and following the guidelines approved by the Institutional Review Board of our hospital.

\section{Consent for publication}

Not applicable. 
Availability of data and materials

All data generated during this research are included in this published manuscript.

\section{Conflict of interest}

The authors declare no conflict of interest.

\section{Funding}

This work was supported by grants from the National Natural Science Foundation of China (Grant Nos. 81801433, 81672648 and 81400664), the CAMS Innovation Fund for Medical Sciences and health (Grant Nos. 2020-PT320-003 and 2016-I2M-1-001), and special funds from the CAMS Molecular Pathology Center and the Central Public Welfare Institutions of CAMS (Nos. 2016ZX310176-4 and 2017PT31008).

\section{Author contributions}

Software, data curation, formal analysis and visualization, HM, PHF, RQH and ZXG; writing-original draft preparation and writing review and editing, $\mathrm{HM}$ and PHF; conceptualization and design, administration, funding acquisition, and critical revision of the manuscript, SNY, JC and QY. All authors read and approved the final manuscript.

\section{Acknowledgments}

We acknowledge the TCGA database for providing their platforms and contributors for uploading their meaningful datasets. The authors would like to thank Editage (www.editage.cn) for English language polishing.

\section{References}

1. Bray, F., J. Ferlay, I. Soerjomataram, R.L. Siegel, L.A. Torre, and A. Jemal, Global cancer statistics 2018: GLOBOCAN estimates of incidence and mortality worldwide for 36 cancers in 185 countries. CA Cancer J Clin, 2018. 68(6): p. 394-424.

2. Siegel, R.L., K.D. Miller, and A. Jemal, Cancer statistics, 2020. CA Cancer J Clin, 2020. 70(1): p. 7-30.

3. Lortet-Tieulent, J., J. Ferlay, F. Bray, and A. Jemal, International Patterns and Trends in Endometrial Cancer Incidence, 1978-2013. J Natl Cancer Inst, 2018. 110(4): p. 354-361.

4. Gordon, J., J.W. Reagan, W.D. Finkle, and H.K. Ziel, Estrogen and endometrial carcinoma. An independent pathology review supporting original risk estimate. N Engl J Med, 1977. 297(11): p. 5701.

5. Quinn, M.J., Secondary analyses from a randomized clinical trial: age as the key prognostic factor in endometrial carcinoma. Am J Obstet Gynecol, 2014. 210(6): p. 588. 
6. Turbiner, J., G. Moreno-Bueno, S. Dahiya, C. Sánchez-Estevez, D. Hardisson, J. Prat, et al., Clinicopathological and molecular analysis of endometrial carcinoma associated with tamoxifen. Mod Pathol, 2008. 21(8): p. 925-36.

7. Kaur, S., J.E. Lotsari, S. Al-Sohaily, J. Warusavitarne, M.R. Kohonen-Corish, and P. Peltomaki, Identification of subgroup-specific miRNA patterns by epigenetic profiling of sporadic and Lynch syndrome-associated colorectal and endometrial carcinoma. Clin Epigenetics, 2015. 7: p. 20.

8. De Angelis, R., M. Sant, M.P. Coleman, S. Francisci, P. Baili, D. Pierannunzio, et al., Cancer survival in Europe 1999-2007 by country and age: results of EUROCARE-5-a population-based study. Lancet Oncol, 2014. 15(1): p. 23-34.

9. Chaudhry, P. and E. Asselin, Resistance to chemotherapy and hormone therapy in endometrial cancer. Endocr Relat Cancer, 2009. 16(2): p. 363-80.

10. Phuengkham, H., L. Ren, I.W. Shin, and Y.T. Lim, Nanoengineered Immune Niches for Reprogramming the Immunosuppressive Tumor Microenvironment and Enhancing Cancer Immunotherapy. Adv Mater, 2019. 31(34): p. e1803322.

11. De Palma, M., D. Biziato, and T.V. Petrova, Microenvironmental regulation of tumour angiogenesis. Nat Rev Cancer, 2017. 17(8): p. 457-474.

12. Tsimberidou, A.M., E. Fountzilas, M. Nikanjam, and R. Kurzrock, Review of precision cancer medicine: Evolution of the treatment paradigm. Cancer Treat Rev, 2020. 86: p. 102019.

13. Prasetyanti, P.R. and J.P. Medema, Intra-tumor heterogeneity from a cancer stem cell perspective. Mol Cancer, 2017. 16(1): p. 41.

14. Driessens, G., B. Beck, A. Caauwe, B.D. Simons, and C. Blanpain, Defining the mode of tumour growth by clonal analysis. Nature, 2012. 488(7412): p. 527-30.

15. Junttila, M.R. and F.J. de Sauvage, Influence of tumour micro-environment heterogeneity on therapeutic response. Nature, 2013. 501(7467): p. 346-54.

16. Hinshaw, D.C. and L.A. Shevde, The Tumor Microenvironment Innately Modulates Cancer Progression. Cancer Res, 2019. 79(18): p. 4557-4566.

17. Ali, H.R., L. Chlon, P.D. Pharoah, F. Markowetz, and C. Caldas, Patterns of Immune Infiltration in Breast Cancer and Their Clinical Implications: A Gene-Expression-Based Retrospective Study. PLoS Med, 2016. 13(12): p. e1002194.

18. Newman, A.M., C.L. Liu, M.R. Green, A.J. Gentles, W. Feng, Y. Xu, et al., Robust enumeration of cell subsets from tissue expression profiles. Nat Methods, 2015. 12(5): p. 453-7.

19. Yoshihara, K., M. Shahmoradgoli, E. Martínez, R. Vegesna, H. Kim, W. Torres-Garcia, et al., Inferring tumour purity and stromal and immune cell admixture from expression data. Nat Commun, 2013. 4: p. 2612.

20. Bindea, G., B. Mlecnik, M. Tosolini, A. Kirilovsky, M. Waldner, A.C. Obenauf, et al., Spatiotemporal dynamics of intratumoral immune cells reveal the immune landscape in human cancer. Immunity, 2013. 39(4): p. 782-95. 
21. Beatty, G.L. and W.L. Gladney, Immune escape mechanisms as a guide for cancer immunotherapy. Clin Cancer Res, 2015. 21(4): p. 687-92.

22. Ciardullo, S., E. Muraca, S. Perra, E. Bianconi, F. Zerbini, A. Oltolini, et al., Screening for non-alcoholic fatty liver disease in type 2 diabetes using non-invasive scores and association with diabetic complications. BMJ Open Diabetes Res Care, 2020. 8(1).

23. George, B., S. Seals, and I. Aban, Survival analysis and regression models. J Nucl Cardiol, 2014. 21(4): p. 686-94.

24. Ritchie, M.E., B. Phipson, D. Wu, Y. Hu, C.W. Law, W. Shi, et al., limma powers differential expression analyses for RNA-sequencing and microarray studies. Nucleic Acids Res, 2015. 43(7): p. e47.

25. Yu, G., L.G. Wang, Y. Han, and Q.Y. He, clusterProfiler: an R package for comparing biological themes among gene clusters. Omics, 2012. 16(5): p. 284-7.

26. Chen, B., D. Wang, J. Li, Y. Hou, and C. Qiao, Screening and Identification of Prognostic TumorInfiltrating Immune Cells and Genes of Endometrioid Endometrial Adenocarcinoma: Based on The Cancer Genome Atlas Database and Bioinformatics. Front Oncol, 2020. 10: p. 554214.

27. Kim, K., S. Jeon, T.M. Kim, and C.K. Jung, Immune Gene Signature Delineates a Subclass of Papillary Thyroid Cancer with Unfavorable Clinical Outcomes. Cancers (Basel), 2018. 10(12).

28. Zong, L., M. Zhang, W. Wang, X. Wan, J. Yang, and Y. Xiang, PD-L1, B7-H3 and VISTA are highly expressed in gestational trophoblastic neoplasia. Histopathology, 2019. 75(3): p. 421-430.

29. Zong, L., S. Mo, S. Yu, Y. Zhou, M. Zhang, J. Chen, et al., Expression of the immune checkpoint VISTA in breast cancer. Cancer Immunol Immunother, 2020. 69(8): p. 1437-1446.

30. Altman, D.G., L.M. McShane, W. Sauerbrei, and S.E. Taube, Reporting Recommendations for Tumor Marker Prognostic Studies (REMARK): explanation and elaboration. PLoS Med, 2012. 9(5): p. e1001216.

31. Remmele, W. and H.E. Stegner, [Recommendation for uniform definition of an immunoreactive score (IRS) for immunohistochemical estrogen receptor detection (ER-ICA) in breast cancer tissue]. Pathologe, 1987. 8(3): p. 138-40.

32. Massarelli, E., V.K. Lam, E.R. Parra, J. Rodriguez-Canales, C. Behrens, L. Diao, et al., High OX-40 expression in the tumor immune infiltrate is a favorable prognostic factor of overall survival in nonsmall cell lung cancer. J Immunother Cancer, 2019. 7(1): p. 351.

33. Fong, L. and E.J. Small, Anti-cytotoxic T-lymphocyte antigen-4 antibody: the first in an emerging class of immunomodulatory antibodies for cancer treatment. J Clin Oncol, 2008. 26(32): p. 5275-83.

34. Malamas, A.S., S.A. Hammond, J. Schlom, and J.W. Hodge, Combination therapy with an OX40L fusion protein and a vaccine targeting the transcription factor twist inhibits metastasis in a murine model of breast cancer. Oncotarget, 2017. 8(53): p. 90825-90841.

35. Kvarnhammar, A.M., N. Veitonmäki, K. Hägerbrand, A. Dahlman, K.E. Smith, S. Fritzell, et al., The CTLA-4 x OX40 bispecific antibody ATOR-1015 induces anti-tumor effects through tumor-directed immune activation. J Immunother Cancer, 2019. 7(1): p. 103. 
36. Bell, R.B., R.S. Leidner, M.R. Crittenden, B.D. Curti, Z. Feng, R. Montler, et al., OX40 signaling in head and neck squamous cell carcinoma: Overcoming immunosuppression in the tumor microenvironment. Oral Oncol, 2016. 52: p. 1-10.

37. Buchan, S.L., A. Rogel, and A. Al-Shamkhani, The immunobiology of CD27 and OX40 and their potential as targets for cancer immunotherapy. Blood, 2018. 131(1): p. 39-48.

38. Liu, Y., L. Patel, G.B. Mills, K.H. Lu, A.K. Sood, L. Ding, et al., Clinical significance of CTNNB1 mutation and Wnt pathway activation in endometrioid endometrial carcinoma. J Natl Cancer Inst, 2014. 106(9).

39. Chen, P., Y. Yang, Y. Zhang, S. Jiang, X. Li, and J. Wan, Identification of prognostic immune-related genes in the tumor microenvironment of endometrial cancer. Aging (Albany NY), 2020. 12(4): p. 3371-3387.

40. Sugamura, K., N. Ishii, and A.D. Weinberg, Therapeutic targeting of the effector T-cell co-stimulatory molecule OX40. Nat Rev Immunol, 2004. 4(6): p. 420-31.

41. Paterson, D.J., W.A. Jefferies, J.R. Green, M.R. Brandon, P. Corthesy, M. Puklavec, et al., Antigens of activated rat $\mathrm{T}$ lymphocytes including a molecule of 50,000 Mr detected only on CD4 positive T blasts. Mol Immunol, 1987. 24(12): p. 1281-90.

42. Ishii, N., T. Takahashi, P. Soroosh, and K. Sugamura, Chapter 3 - OX40-OX40 Ligand Interaction in TCell-Mediated Immunity and Immunopathology, in Advances in Immunology, F.W. Alt, Editor. 2010, Academic Press. p. 63-98.

43. Jahan, N., H. Talat, and W.T. Curry, Agonist OX40 immunotherapy improves survival in gliomabearing mice and is complementary with vaccination with irradiated GM-CSF-expressing tumor cells. Neuro Oncol, 2018. 20(1): p. 44-54.

44. Fu, Y., Q. Lin, Z. Zhang, and L. Zhang, Therapeutic strategies for the costimulatory molecule OX40 in T-cell-mediated immunity. Acta Pharm Sin B, 2020. 10(3): p. 414-433.

45. Bulliard, Y., R. Jolicoeur, J. Zhang, G. Dranoff, N.S. Wilson, and J.L. Brogdon, OX40 engagement depletes intratumoral Tregs via activating FcyRs, leading to antitumor efficacy. Immunol Cell Biol, 2014. 92(6): p. 475-80.

46. Cebada, J., M. Perez-Santos, C. Bandala, E. Lara-Padilla, I. Herrera-Camacho, N.H. Rosas-Murrieta, et al., OX40 agonists for cancer treatment: a patent review. Expert Opin Ther Pat, 2021. 31(1): p. 81-90.

47. Marabelle, A., H. Kohrt, I. Sagiv-Barfi, B. Ajami, R.C. Axtell, G. Zhou, et al., Depleting tumor-specific Tregs at a single site eradicates disseminated tumors. J Clin Invest, 2013. 123(6): p. 2447-63.

48. Sedwick, C., OX40: a win-win path to tumor immunity. J Exp Med. 2008 Apr 14;205(4):742. doi: 10.1084/jem.2054iti2.

49. Castro, F., A.P. Cardoso, R.M. Gonçalves, K. Serre, and M.J. Oliveira, Interferon-Gamma at the Crossroads of Tumor Immune Surveillance or Evasion. Front Immunol, 2018. 9: p. 847.

50. Rearden, R., A. Sah, B. Doff, T. Kobayashi, S.J. McKee, G.R. Leggatt, et al., Control of B-cell lymphoma by therapeutic vaccination and acquisition of immune resistance is independent of direct tumour IFN-gamma signalling. Immunol Cell Biol, 2016. 94(6): p. 554-62. 
51. de Jong, R.A., N. Leffers, H.M. Boezen, K.A. ten Hoor, A.G. van der Zee, H. Hollema, et al., Presence of tumor-infiltrating lymphocytes is an independent prognostic factor in type I and II endometrial cancer. Gynecol Oncol, 2009. 114(1): p. 105-10.

52. Zheng, C., L. Zheng, J.K. Yoo, H. Guo, Y. Zhang, X. Guo, et al., Landscape of Infiltrating T Cells in Liver Cancer Revealed by Single-Cell Sequencing. Cell, 2017. 169(7): p. 1342-1356.e16.

53. Zhou, R., J. Zhang, D. Zeng, H. Sun, X. Rong, M. Shi, et al., Immune cell infiltration as a biomarker for the diagnosis and prognosis of stage I-III colon cancer. Cancer Immunol Immunother, 2019. 68(3): p. 433-442.

54. Klemm, F., R.R. Maas, R.L. Bowman, M. Kornete, K. Soukup, S. Nassiri, et al., Interrogation of the Microenvironmental Landscape in Brain Tumors Reveals Disease-Specific Alterations of Immune Cells. Cell, 2020. 181(7): p. 1643-1660.e17.

55. Xu, C., S. Sui, Y. Shang, Z. Yu, J. Han, G. Zhang, et al., The landscape of immune cell infiltration and its clinical implications of pancreatic ductal adenocarcinoma. J Adv Res, 2020. 24: p. 139-148.

56. Noël, G., M. Langouo Fontsa, and K. Willard-Gallo, The impact of tumor cell metabolism on T cellmediated immune responses and immuno-metabolic biomarkers in cancer. Semin Cancer Biol, 2018. 52(Pt 2): p. 66-74.

57. Zander, R., D. Schauder, G. Xin, C. Nguyen, X. Wu, A. Zajac, et al., CD4(+) T Cell Help Is Required for the Formation of a Cytolytic CD8(+) T Cell Subset that Protects against Chronic Infection and Cancer. Immunity, 2019. 51(6): p. 1028-1042.e4.

58. Ladányi, A., B. Somlai, K. Gilde, Z. Fejös, I. Gaudi, and J. Tímár, T-cell activation marker expression on tumor-infiltrating lymphocytes as prognostic factor in cutaneous malignant melanoma. Clin Cancer Res, 2004. 10(2): p. 521-30.

59. Petty, J.K., K. He, C.L. Corless, J.T. Vetto, and A.D. Weinberg, Survival in human colorectal cancer correlates with expression of the T-cell costimulatory molecule OX-40 (CD134). Am J Surg, 2002. 183(5): p. 512-8.

60. Ramser, M., S. Eichelberger, S. Däster, B. Weixler, M. Kraljević, R. Mechera, et al., High OX40 expression in recurrent ovarian carcinoma is indicative for response to repeated chemotherapy. BMC Cancer, 2018. 18(1): p. 425.

61. Ohmura, H., K. Yamaguchi, F. Hanamura, M. Ito, A. Makiyama, K. Uchino, et al., OX40 and LAG3 are associated with better prognosis in advanced gastric cancer patients treated with anti-programmed death-1 antibody. Br J Cancer, 2020. 122(10): p. 1507-1517.

62. Xie, K., L. Xu, H. Wu, H. Liao, L. Luo, M. Liao, et al., OX40 expression in hepatocellular carcinoma is associated with a distinct immune microenvironment, specific mutation signature, and poor prognosis. Oncoimmunology, 2018. 7(4): p. e1404214.

63. Speiser, D.E., P.C. Ho, and G. Verdeil, Regulatory circuits of T cell function in cancer. Nat Rev Immunol, 2016. 16(10): p. 599-611.

64. Curti, B.D., M. Kovacsovics-Bankowski, N. Morris, E. Walker, L. Chisholm, K. Floyd, et al., OX40 is a potent immune-stimulating target in late-stage cancer patients. Cancer Res, 2013. 73(24): p. 7189- 
7198.

65. Sagiv-Barfi, I., D.K. Czerwinski, S. Levy, I.S. Alam, A.T. Mayer, S.S. Gambhir, et al., Eradication of spontaneous malignancy by local immunotherapy. Sci Transl Med, 2018. 10(426).

66. Weinberg, A.D., D.E. Evans, C. Thalhofer, T. Shi, and R.A. Prell, The generation of T cell memory: a review describing the molecular and cellular events following OX40 (CD134) engagement. J Leukoc Biol, 2004. 75(6): p. 962-72.

\section{Figures}




\section{TCGA-UCEC Dataset Acquistion and Processing}

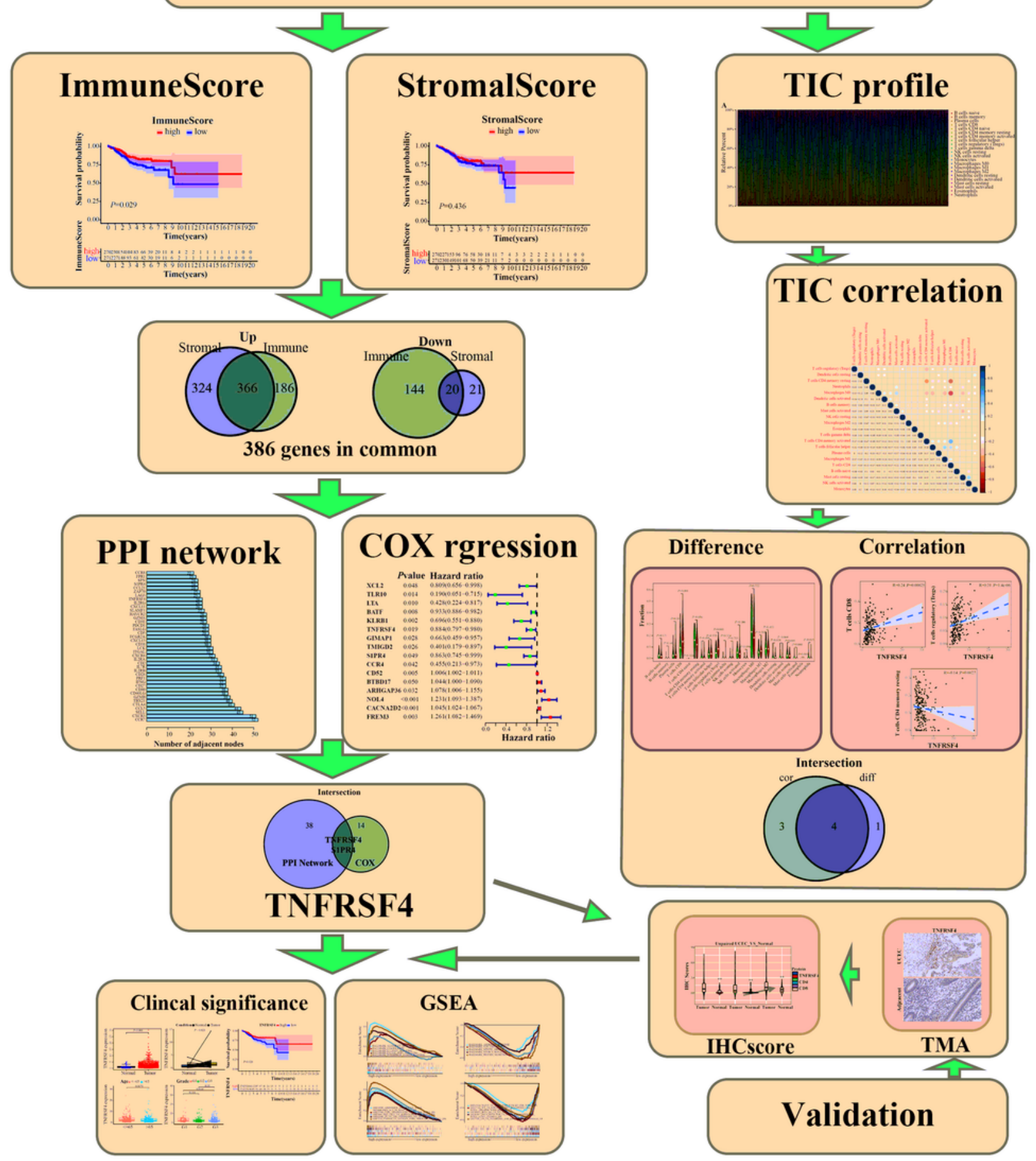

Figure 1

The flow diagram of the research design. This flow chart presented a comprehensive bioinformatics analysis and cohort validation to screen out the putative target gene, TNFRSF4, and investigate its clinicopathologic significance in UCEC. 
A

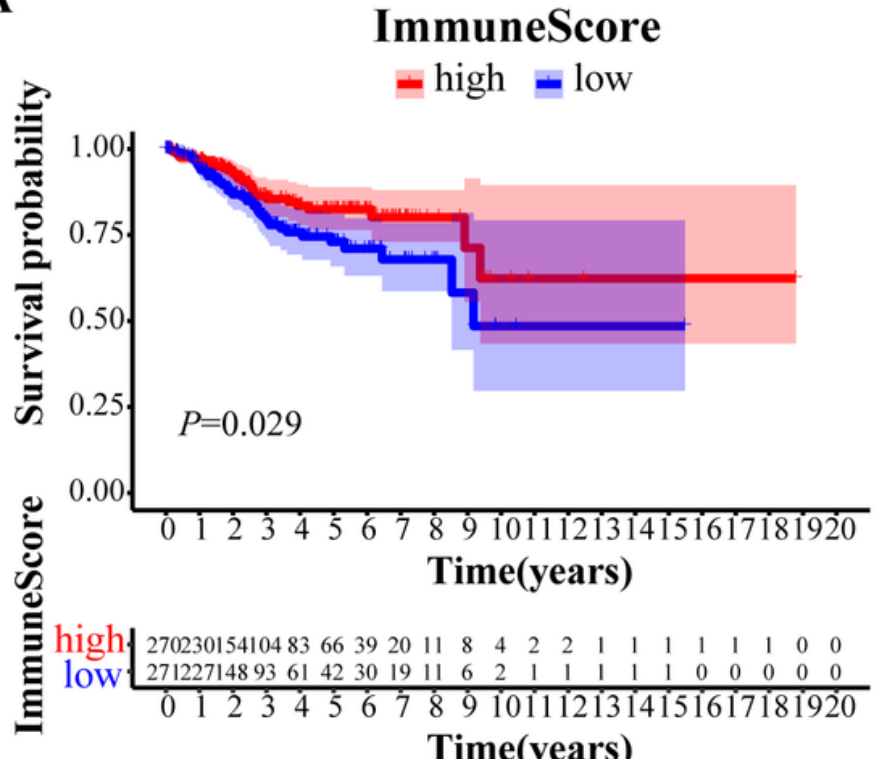

B

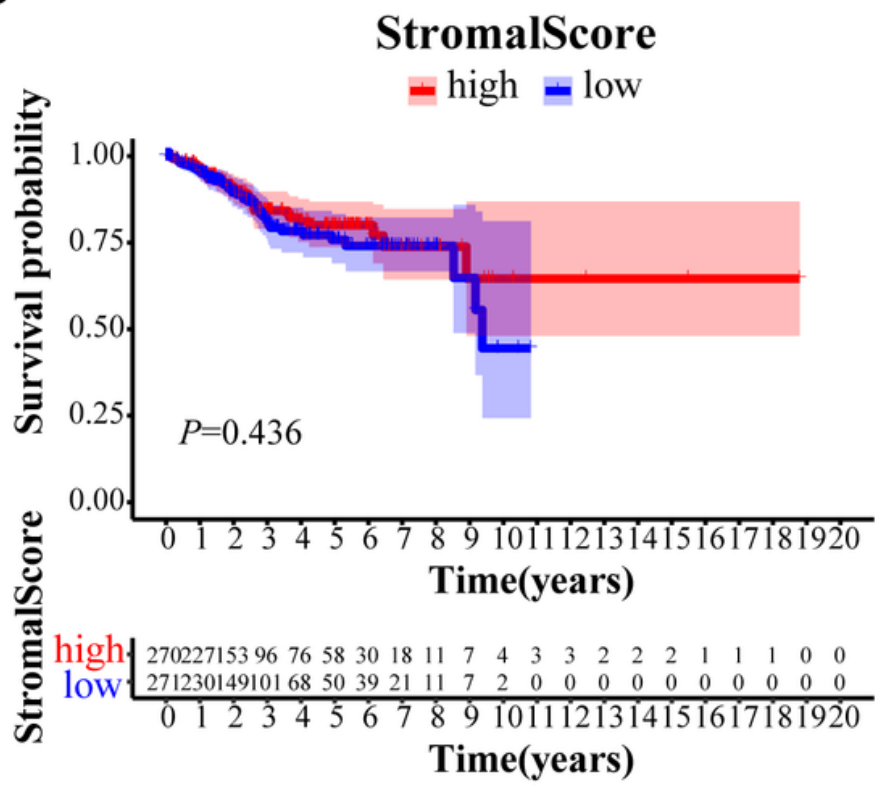

C

ESTIMATEScore

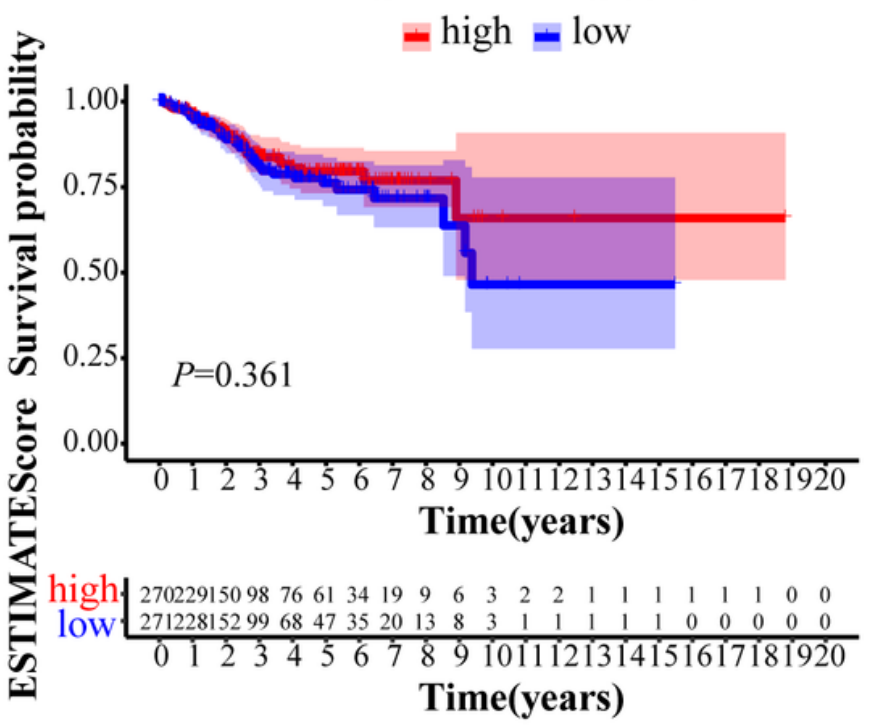

\section{Figure 2}

Survival probability correlated with Estimate scores. A. Comparison of the survival rate between the high or low ImmuneScore groups in UCEC $(P=0.029, n=541)$. B. Kaplan-Meier survival for UCEC patients with high or low StromalScore $(P=0.436, n=541)$. C. Comparison of the survival rate between the high or low ESTIMATEScore groups in UCEC by log-rank test $(P=0.361, n=541)$. 
A

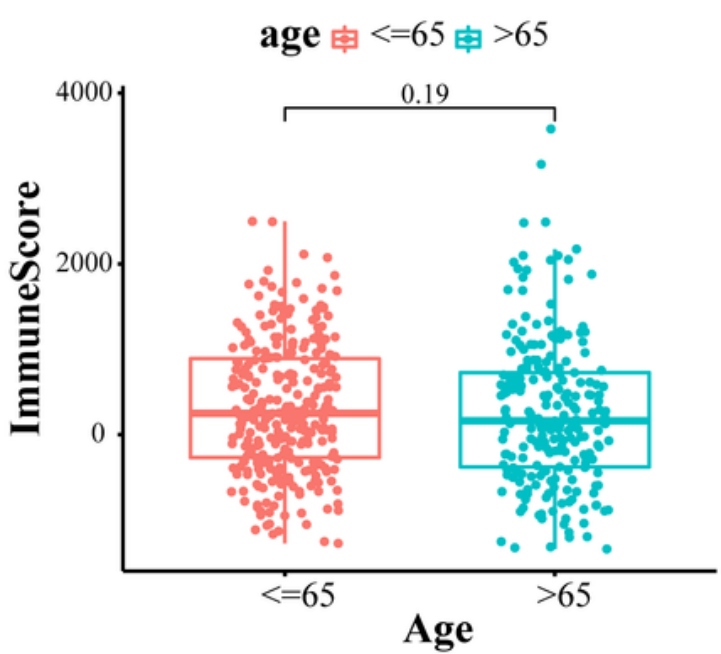

C

$$
\text { age 审 }<=65 \text { 审 }>65
$$

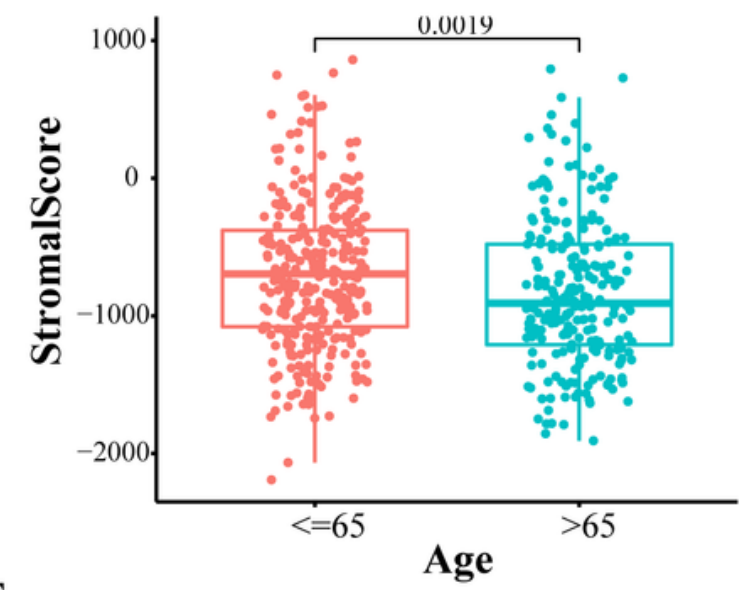

$\mathbf{E}$

$$
\text { age } \text { 审 }<=65 \text { 审 }>65
$$

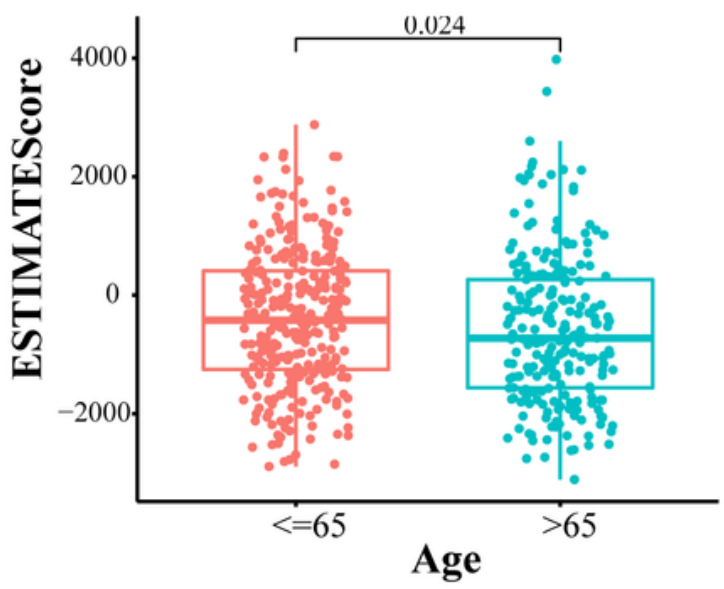

B

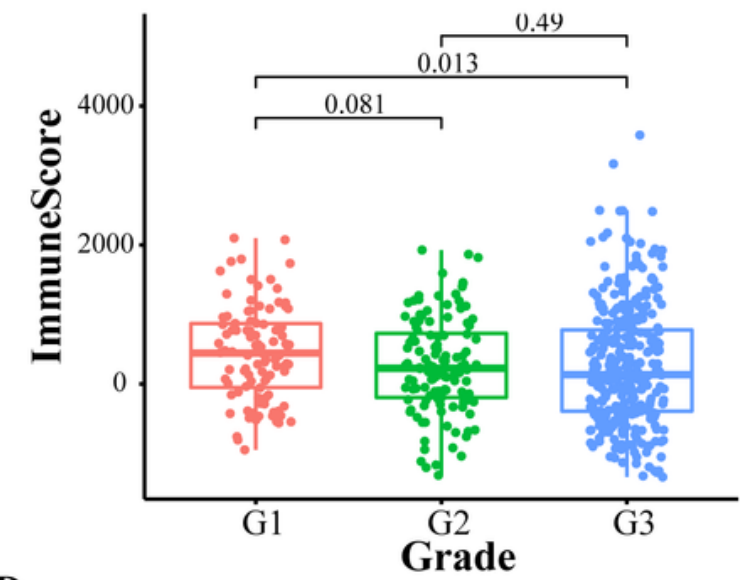

D

grade 审 $\mathrm{G} 1$ 审 $\mathrm{G} 2$ 审 $\mathrm{G} 3$

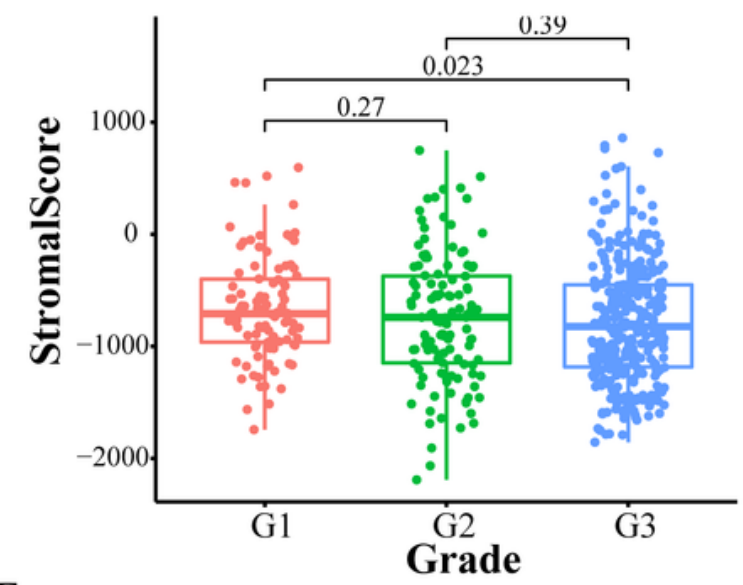

$\mathbf{F}$

grade 审 $\mathrm{G} 1$ 审 $\mathrm{G} 2$ 审 $\mathrm{G} 3$

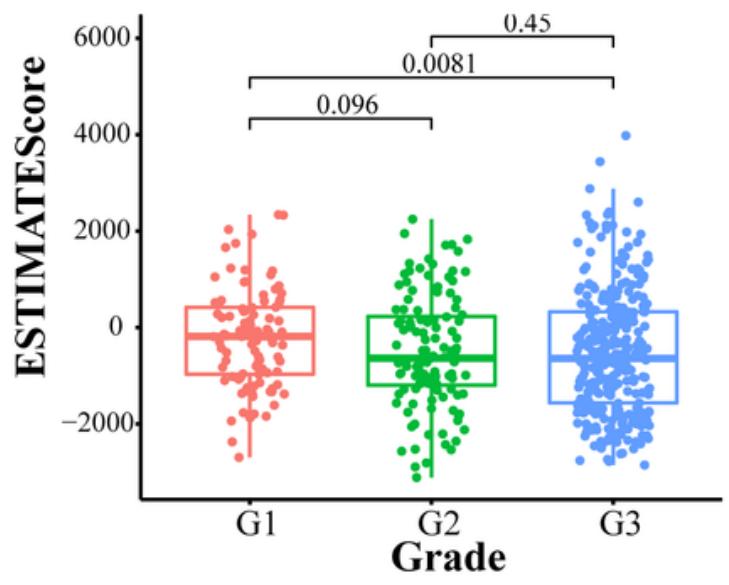

\section{Figure 3}

Correlation between Estimate scores with clinicopathologic parameters. A, C, E. Distribution of ImmuneScore, StromalScore, and ESTIMATEScore grouped by age $(P=0.19,0.0019,0.024$, respectively). B, D, F. Distribution of ImmuneScore, StromalScore, and ESTIMATEScore grouped by grade of UCEC. Comparisons were performed by the Kruskal Wallis rank-sum test. 
A
B

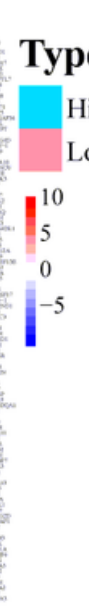

StromalScore

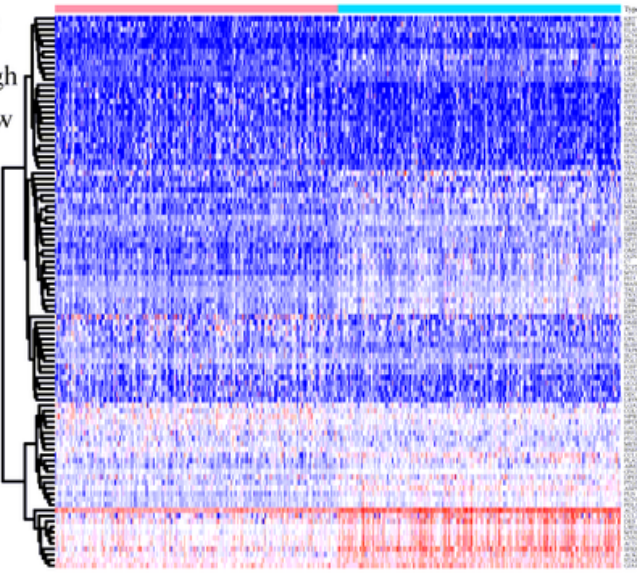

C

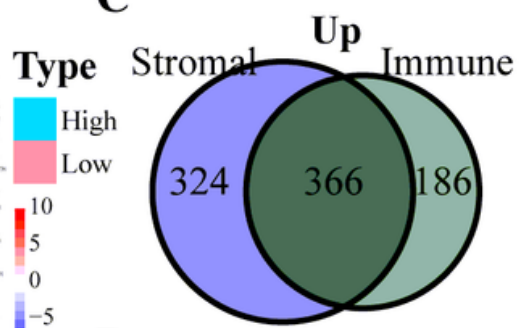

D

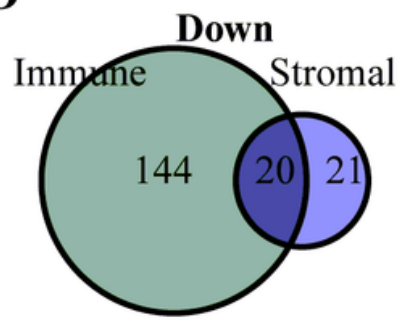

E

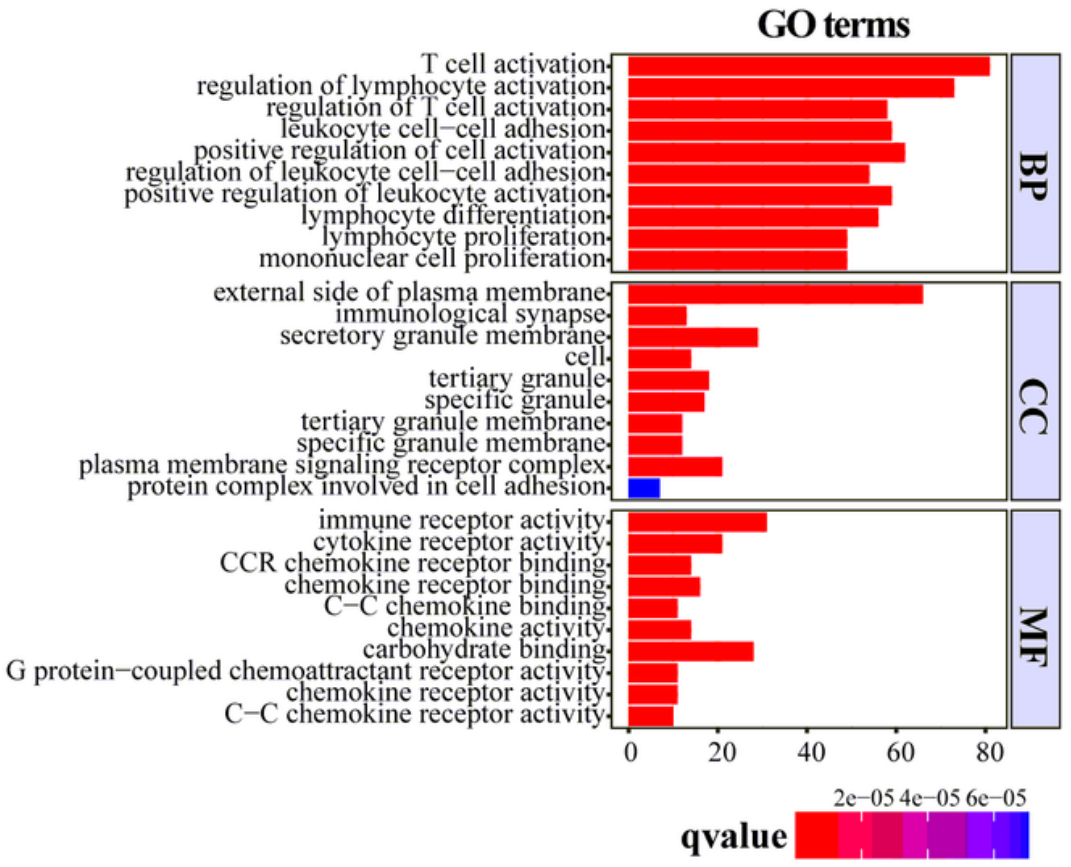

$\mathbf{F}$

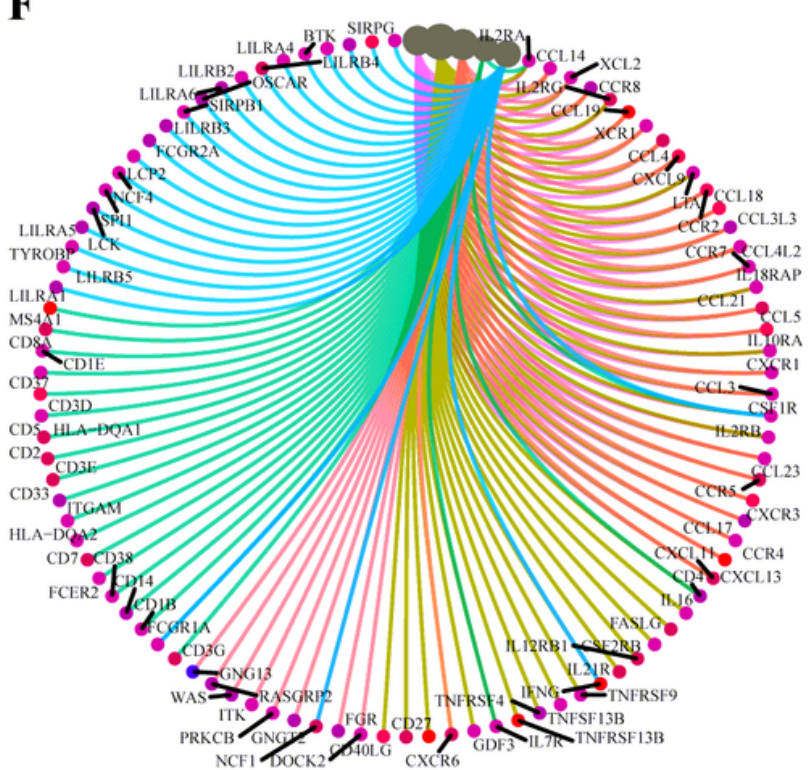

\section{Category}

- Chemokine signaling pathway

- Cytokine-cytokine receptor interaction - Hematopoietic cell lineage - Osteoclast differentiation

- Viral protein interaction with cytokine and cytokine receptor

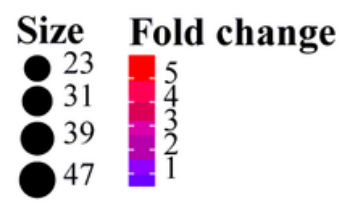

\section{Figure 4}

Screening of differentially expressed genes and functional enrichment. A. The top 50 differentially expressed genes of UCEC grouped by ImmuneScore, displayed by heatmap. Rownames represented the gene names, the clustering characteristics of which were analyzed according to the expression patterns of DEGs. B. Heatmap of the top 50 differentially expressed genes generated by comparison between high or low StromalScore groups, with FDR $<0.05$ and $|\log F C|>1$. Color stood for the expression level of DEGs. C, D. Genes shared by ImmuneScore and StromalScore, displayed by Venn plots (Upper panel for the up-regulated genes and lower panel for the down-regulated ones). E. Bar plots for the top 10 enriched terms of biological process, cellular component, and molecular function of 386 DEGs in GO enrichment, 
respectively, where q value $<0.05$ were regarded to be enriched significantly. F. Circos plot for functional classification of KEGG with the identified categories displayed.

A

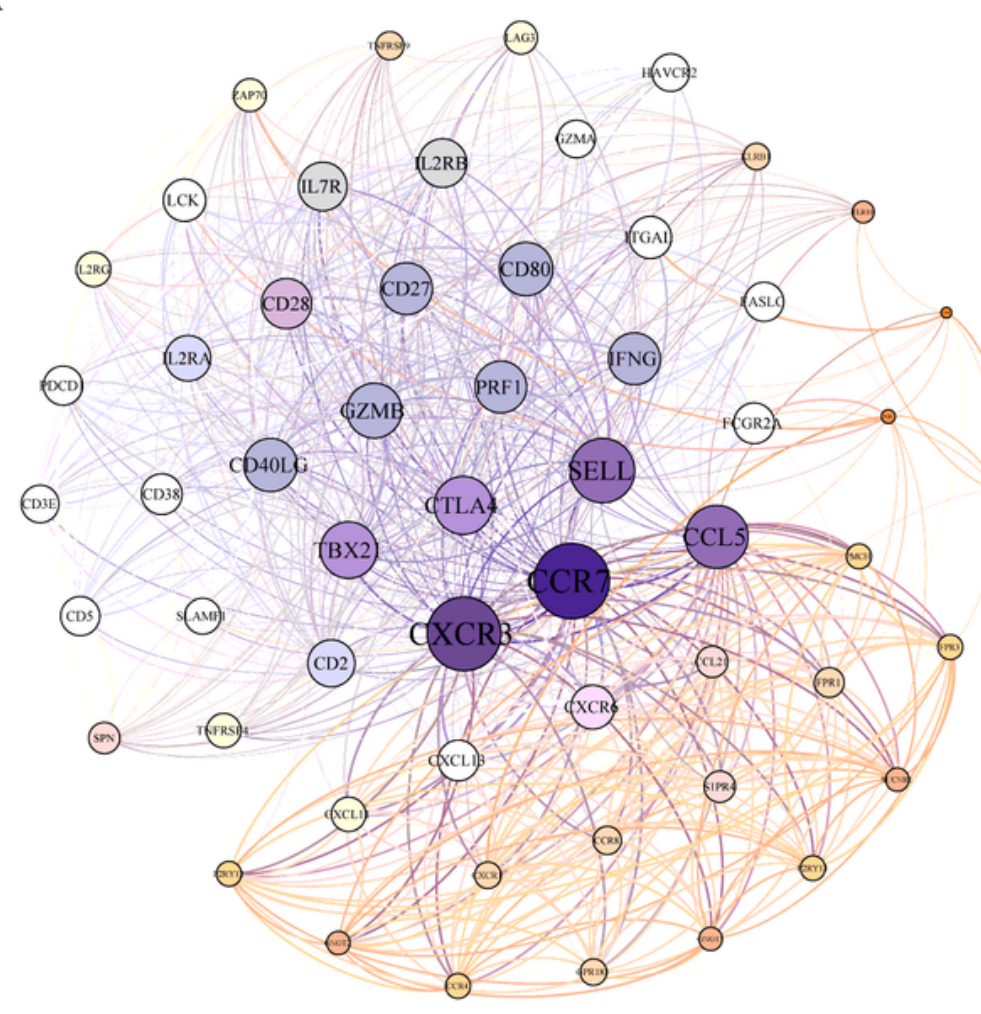

C

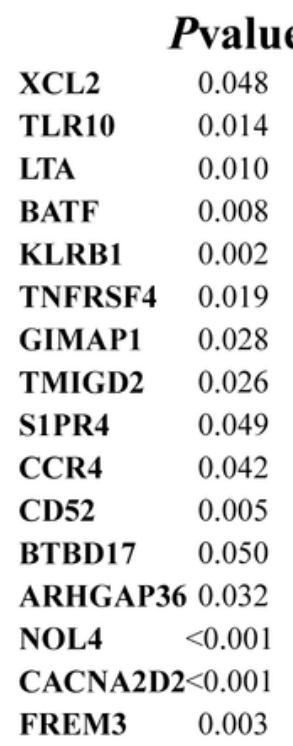

B

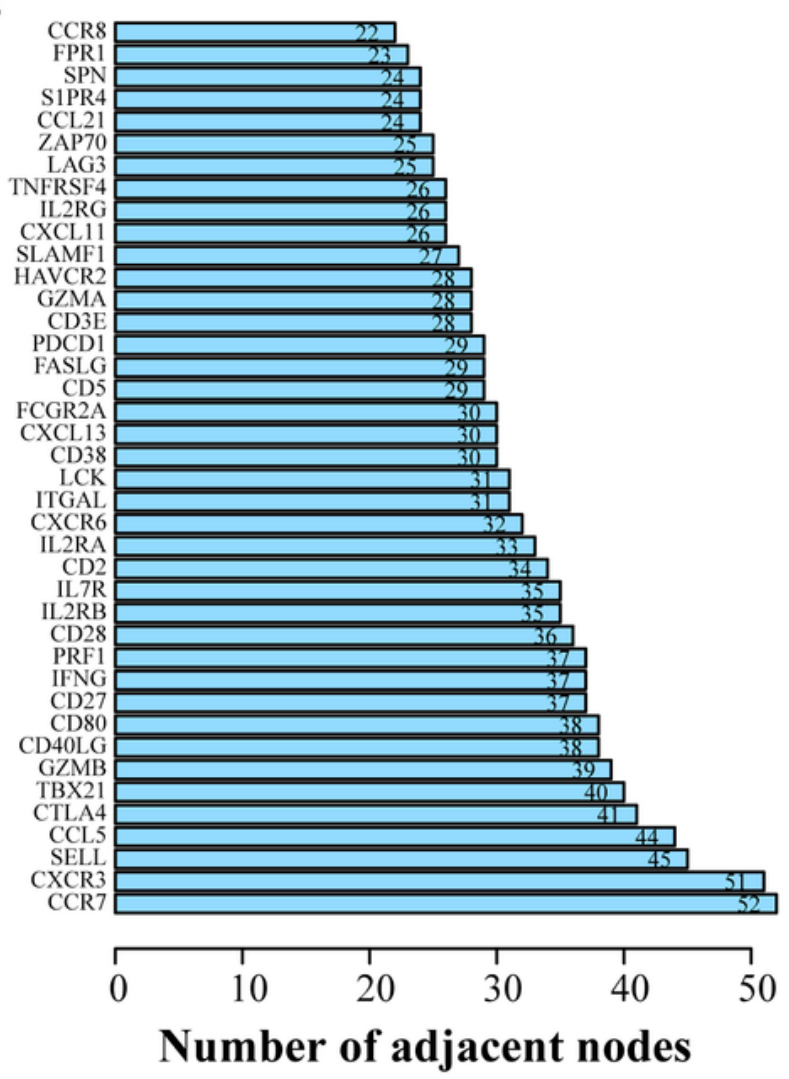

D

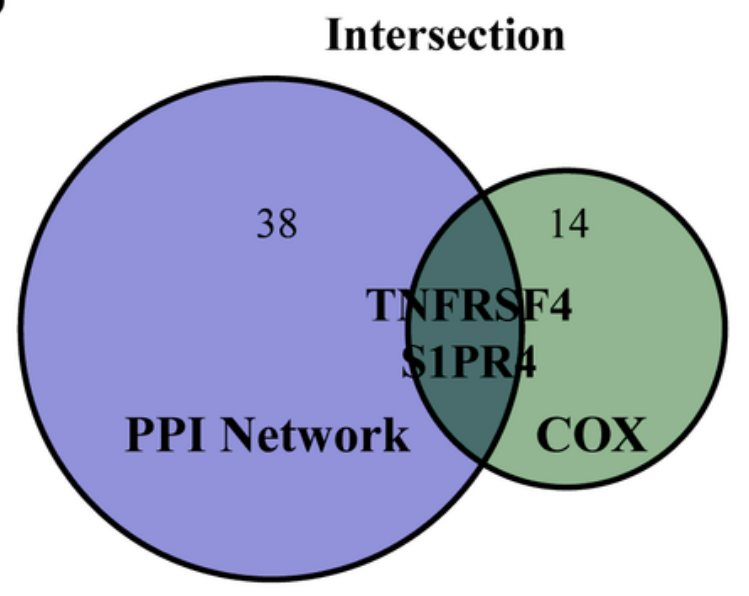

\section{Figure 5}

PPI network and univariate Cox regression analysis to determine the target gene. A. The interactive network of hub cluster, filtered by MCODE analysis. Each node represented a gene. Edges among genes symbolized the interconnected relationships among genes. 56 genes were involved in the cluster. B. The 
top 40 genes ordered by the number of nodes. C. Univariate Cox regression analysis with $386 \mathrm{DEGs}$, correlated with survival probability in UCEC with $P<0.05$. D. Overlapped genes, shared by the top 40 genes in PPI and 16 prognosis-related genes.

A

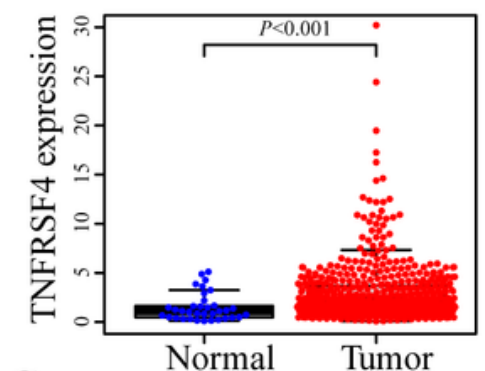

C

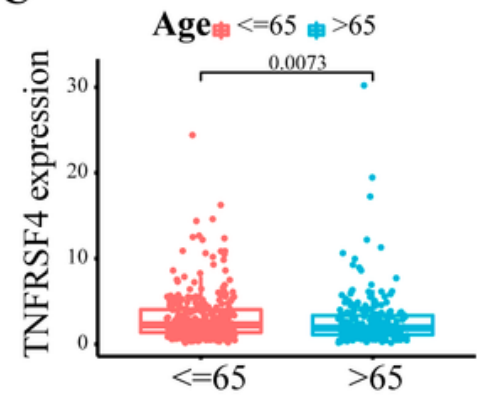

F

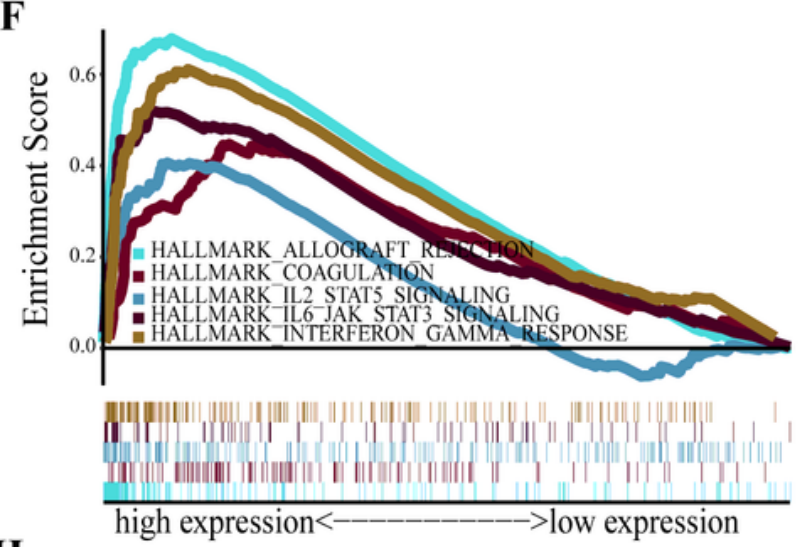

$\mathbf{H}$

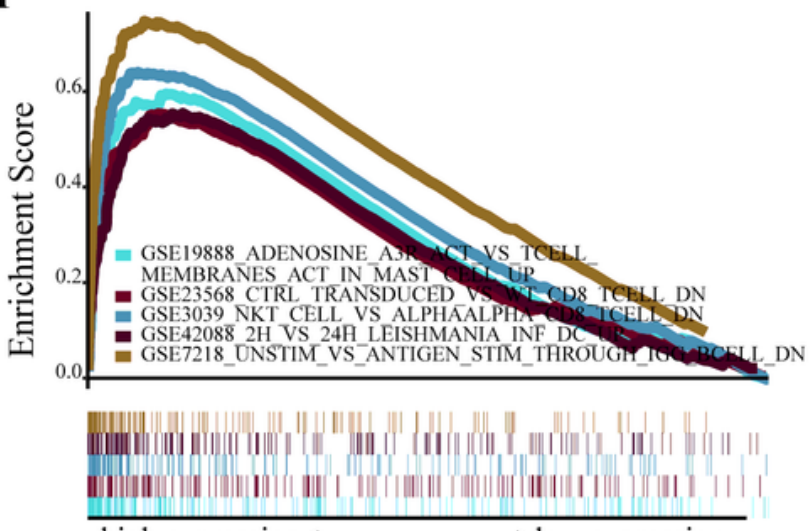

high expression<----------> low expression
B Condition Normal Tumor

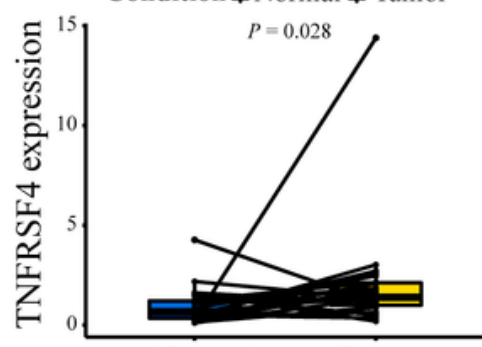

D Normal Tumor

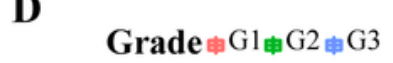

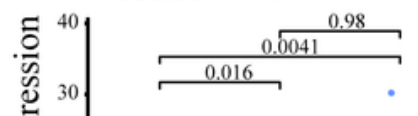

.

,
$\mathbf{E}$

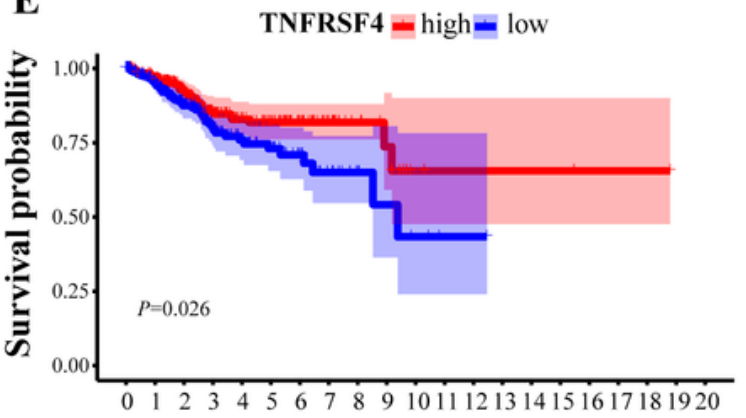

Time(years)

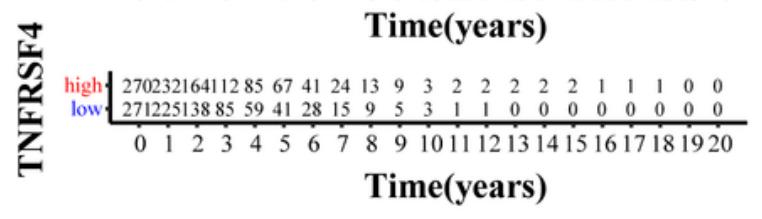

\section{G \\ G}

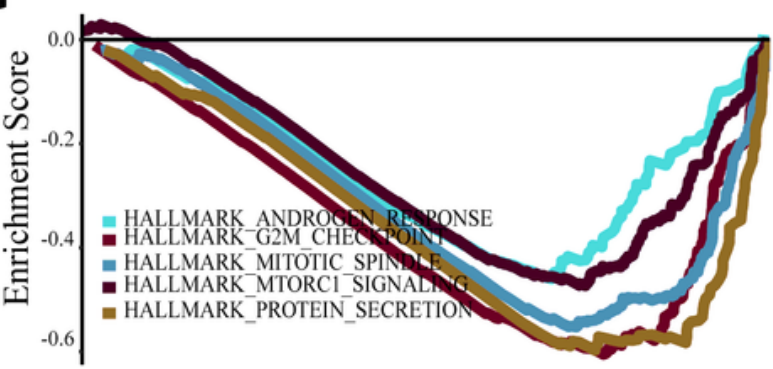

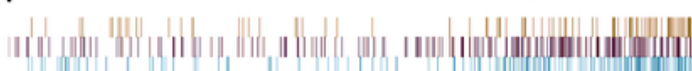

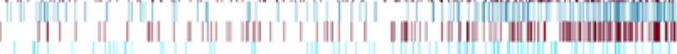

high expression<----------->low expression

I

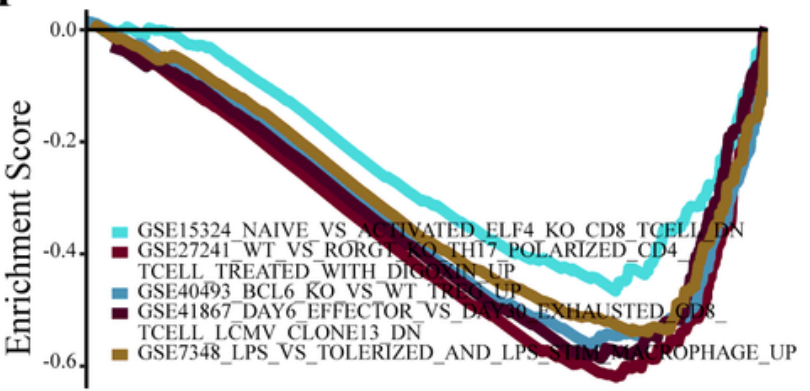

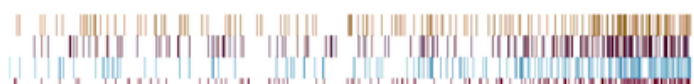

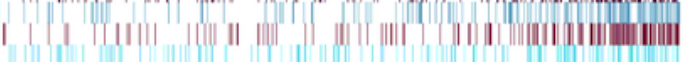

high expression<------------>low expression

\section{Figure 6}

The expression level and GSEA analysis of TNFRSF4 and its clinicopathologic significance. A. Expression level of TNFRSF4 in the normal and tumor tissues $(P<0.001)$. B. Differential analysis for paired samples 
to detect the expression of TNFRSF4. The dots were connected by lines on behalf of pairs of samples from either normal or tumor tissues from a common patient. C. Differential expression of TNFRSF4 of samples, grouped by age. D. The correlation of the expression of TNFRSF4 compared among groups classified by pathological grade using the Kruskal Wallis rank-sum test. E. Survival probability analysis for UCEC patients with different TNFRSF4 expression. Patients were recognized as high or low expression according to the comparison with the median expression level. $P=0.026$. $F$. The involved gene sets in HALLMARK collection by the high TNFRSF4 expression sample. The line chart of the upper part represented the enrichment score of the gene sets. Each line described one particular gene set, distinguished by a unique color and five leading gene sets were displayed in the plot. G. The enriched gene sets in HALLMARK by samples with low TNFRSF4 expression. H. Enriched gene sets in immunologic signatures by samples of high TNFRSF4 expression. I. Enriched gene sets in immunologic signatures by the low TNFRSF4 expression. 


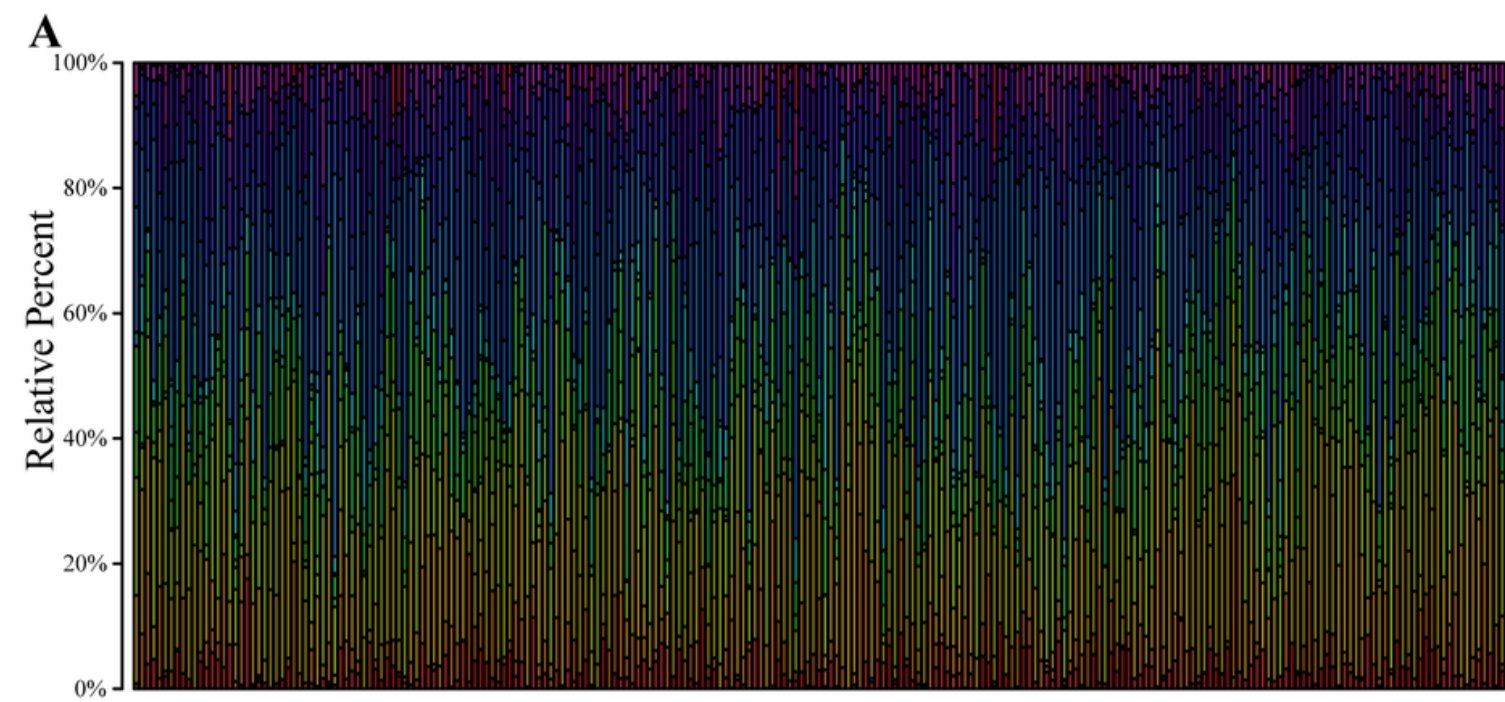

- B cells naive - B cells memory

- Plasma cells

- T cells CD8

- T cells CD4 naive

- T cells CD4 memory resting

- T cells CD4 memory activated

- T cells follicular helper

" T cells regulatory (Tregs)

- T cells gamma delta

- NK cells resting

- NK cells activated

- Monocytes

- Macrophages M0

- Macrophages M1

- Macrophages M2

- Dendritic cells resting

- Dendritic cells activated

- Mast cells resting

- Mast cells activated

" Eosinophils

- Neutrophils

B

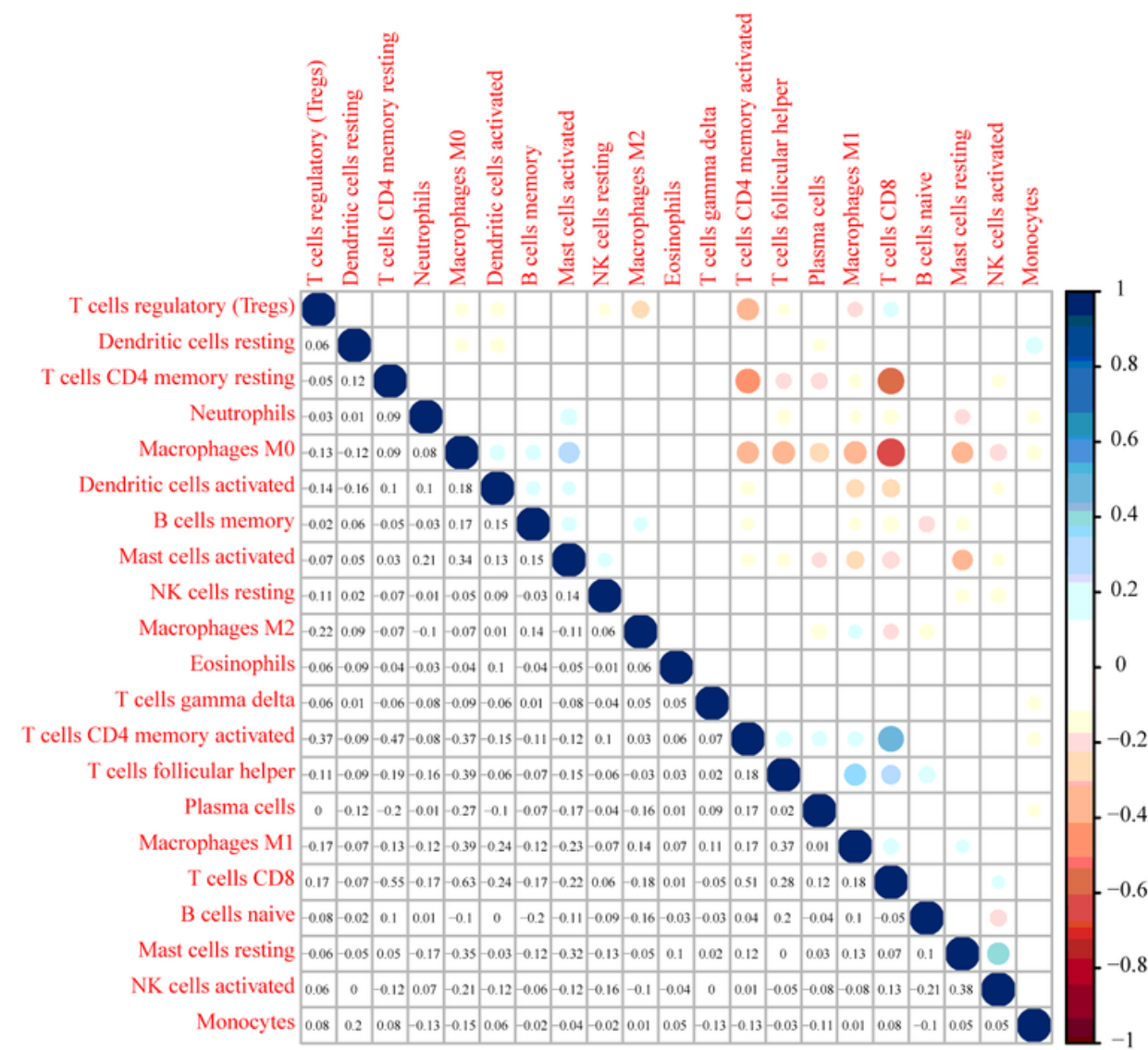

Figure 7

TIC profile in UCEC samples and correlation analysis. A. Bar plot described the component of 22 types of TICs in UCEC tumor samples. Each color represented a kind of immune cell. B. Heatmap showed the correlation among TICs and color and sizes of each dot indicated correlation coefficient between two kinds of cells. 
A
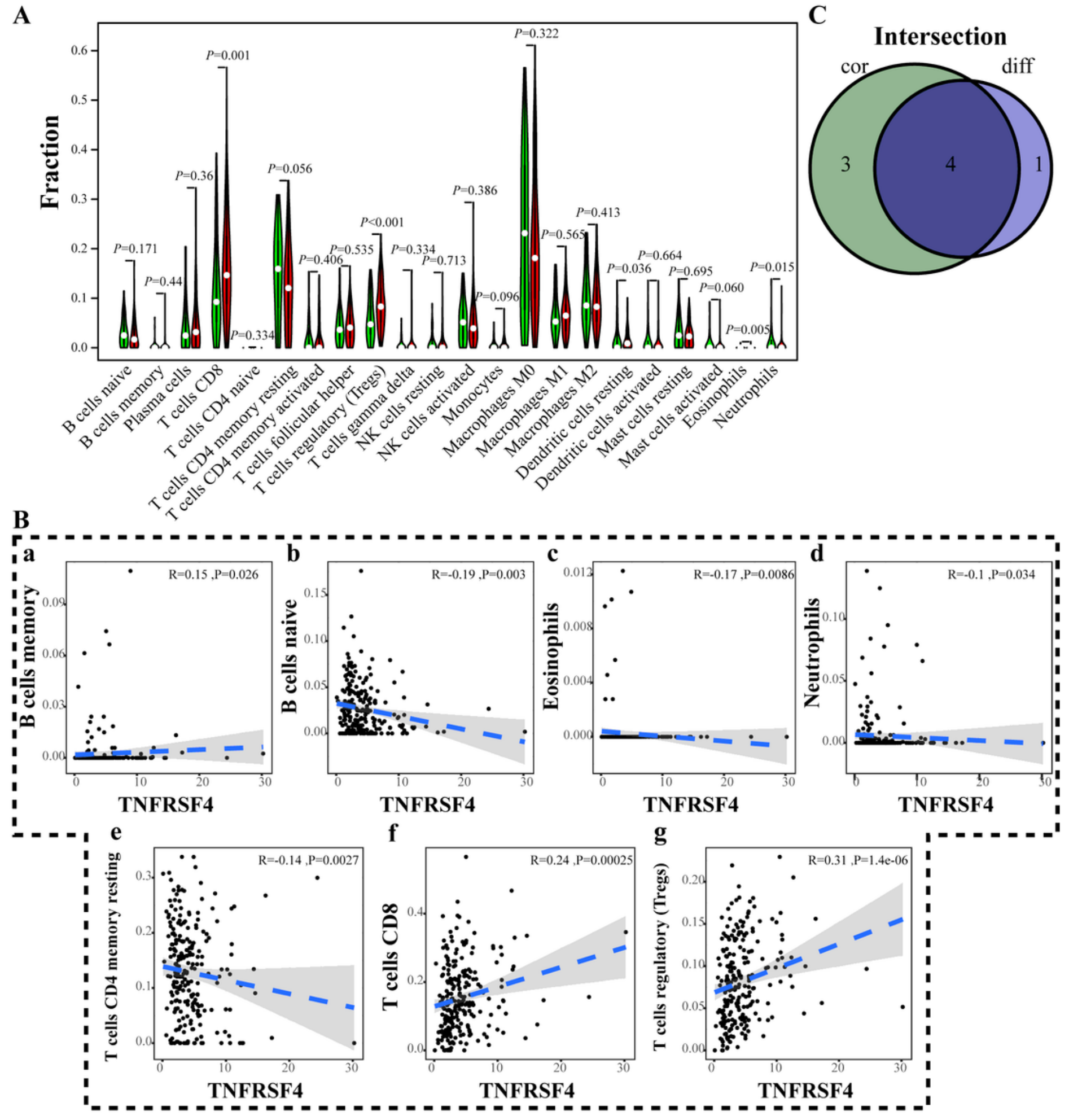

Figure 8

Difference and correlation of TICs proportion with TNFRSF4 expression. A. Violin plot showed the percentage differentiation of 22 types of immune cells among UCEC tumor samples, compared between the high or low-TNFRSF4 expression groups. B. Scatter plots showed the correlation of seven kinds of TICs proportion with the TNFRSF4 expression $(P<0.05)$. The blue line in each plot was a linear fit suggesting the proportion trend of the immune cell as TNFRSF4 expression altered. C. Venn plot 
displayed four kinds of TICs affected by TNFRSF4 expression, co-determined by difference and correlation analysis as displayed in violin and scatter plots.

A

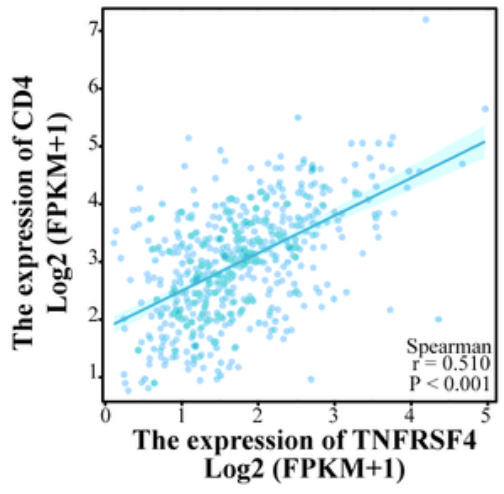

D
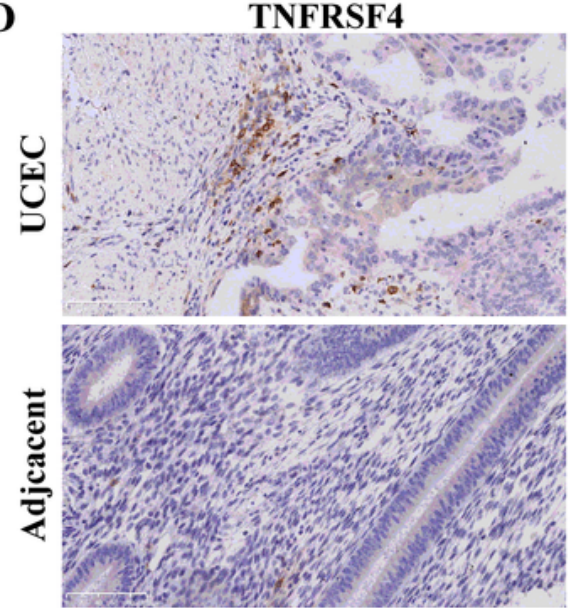

$\mathbf{E}$
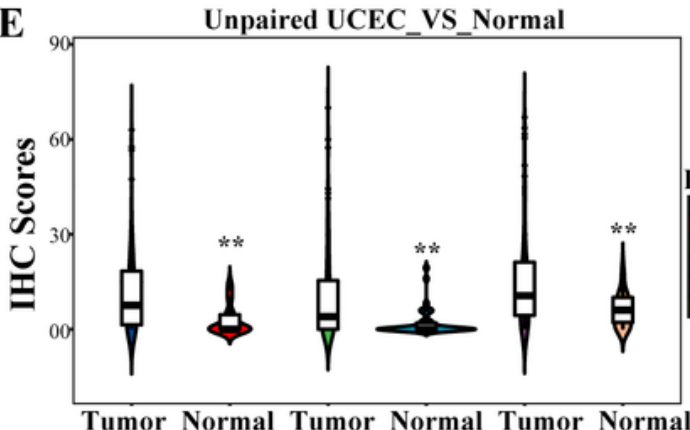

B

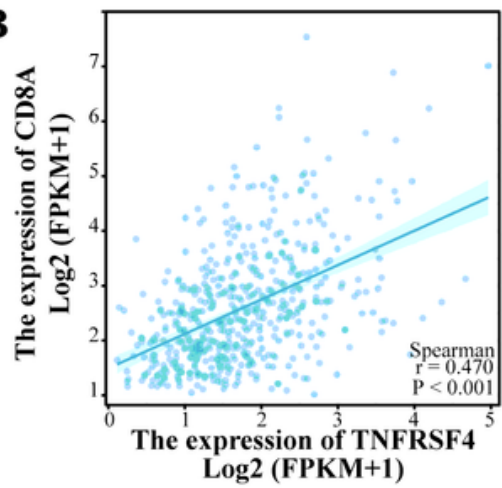

CD4

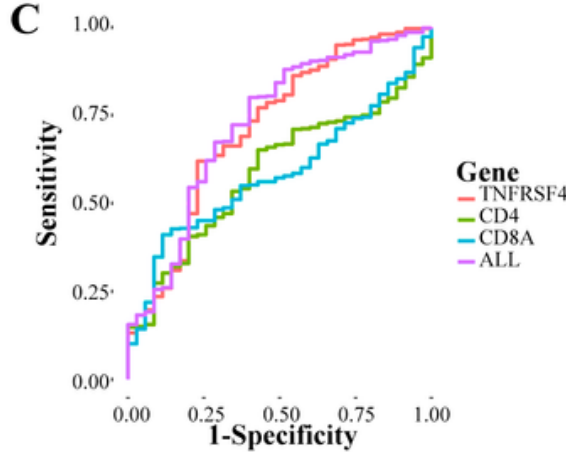

CD8

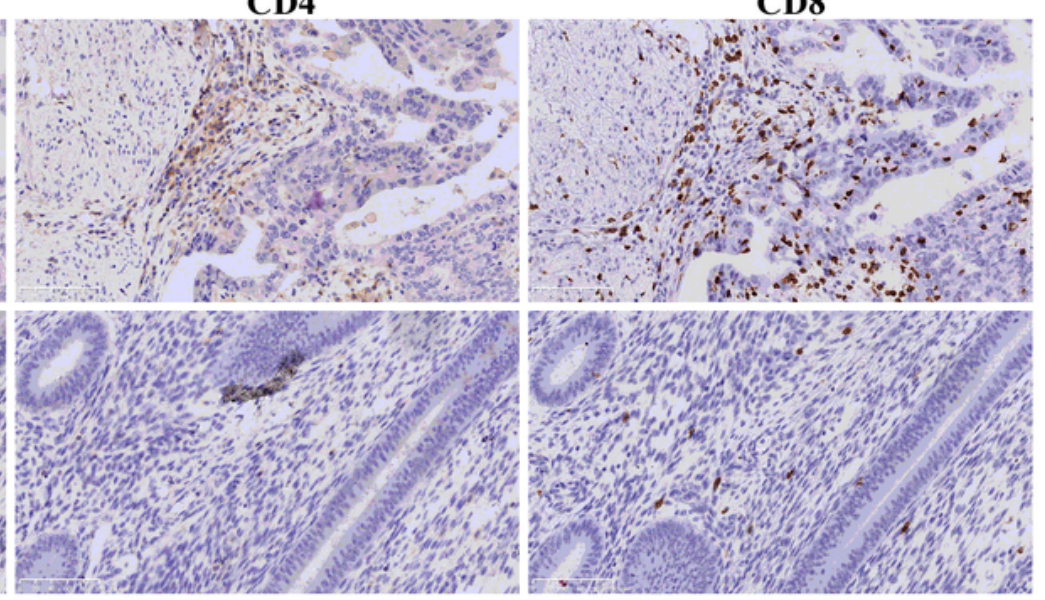

$\mathbf{F}$

F Paired UCEC_VS_Normal
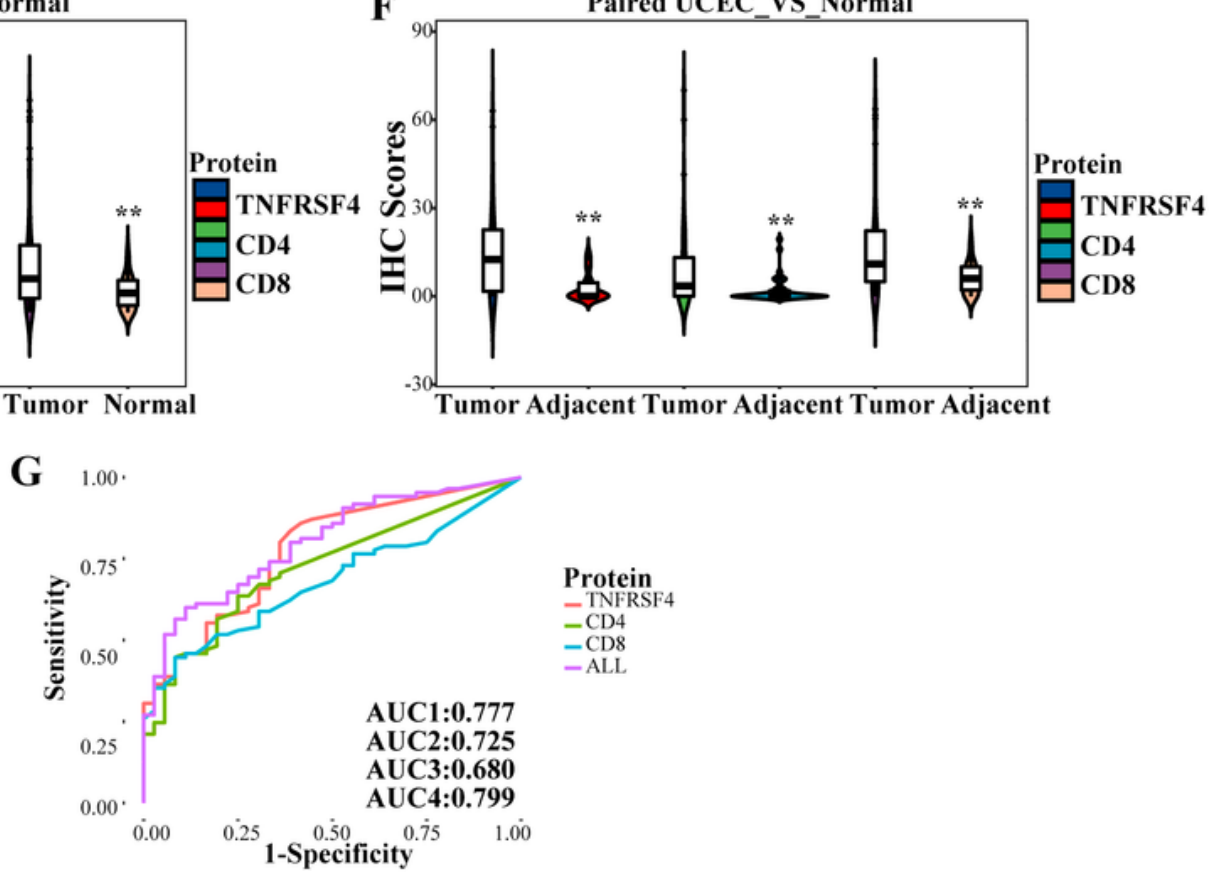

Figure 9

Identification of target genes for correlation, expression, and diagnostic performance in UCEC. A, B. Correlation of TNFRSF4 with CD4 and CD8A. C. ROC curves analyzing the diagnostic performance of TNFRSF4, CD4 and CD8A for UCEC patients in the TCGA. D. Representative IHC stating for TNFRSF4, CD4 
and CD8 in UCEC and adjacent normal tissue. $\times 200$ magnification (scale bar $=100 \mu \mathrm{m}) . \mathrm{E}, \mathrm{F}$. Violin plots visualizing the quantification of TNFRSF4, CD 4 and CD8 in UCEC and adjacent normal tissue. G. ROC curves validating the diagnostic performance of TNFRSF4, CD4 and CD8 in UCEC clinical specimens. 